\title{
Is the full susceptibility of the square-lattice Ising model a differentially algebraic function?
}

\author{
A. J. Guttmann ${ }^{\ddagger}$, I. Jensen ${ }^{\ddagger}$, J-M. Maillard ${ }^{\dagger}$, J. Pantone ${ }^{£}$ \\ $\ddagger$ School of Mathematics and Statistics, The University of Melbourne, Victoria \\ 3010, Australia \\ † LPTMC, UMR 7600 CNRS, Université de Paris 6, Tour 23, 5ème étage, case \\ 121, 4 Place Jussieu, 75252 Paris Cedex 05, France \\ $£$ Department of Mathematics, Dartmouth College, Hanover, NH 03755, USA
}

E-mail: guttmann@unimelb.edu.au,ij@unimelb.edu.au, maillard@lptmc.jussieu.fr, jay.pantone@gmail.com

\begin{abstract}
.
We study the class of non-holonomic power series with integer coefficients that reduce, modulo primes, or powers of primes, to algebraic functions. In particular we try to determine whether the susceptibility of the square-lattice Ising model belongs to this class, and more broadly whether the susceptibility is a solution of a differentially algebraic equation.

Initial results on Tutte's non-linear ordinary differential equation (ODE) and other simple quadratic non-linear ODEs suggest that a large set of differentially algebraic power series solutions with integer coefficients might reduce to algebraic functions modulo primes, or powers of primes. Since diagonals of rational functions are well-known to reduce, modulo primes, or powers of primes, to algebraic functions, a large subset of differentially algebraic power series with integer coefficients may be viewed as a natural "non-linear" generalisation of diagonals of rational functions.

Here we give several examples of series with integer coefficients and nonzero radius of convergence that reduce to algebraic functions modulo (almost) every prime (or power of a prime). These examples satisfy differentially algebraic equations with the encoding polynomial occasionally possessing quite high degree (and thus difficult to identify even with long series). These examples shed important light on the very nature of such differentially algebraic series.

Additionally, we have extended both the high- and low-temperature Ising square-lattice susceptibility series to 5043 coefficients. We find that even this long series is insufficient to determine whether it reduces to algebraic functions modulo 3,5 , etc. This negative result is in contrast to the comparatively easy confirmation that the corresponding series reduce to algebraic functions modulo powers of 2 .

Finally we show that even with 5043 terms we are unable to identify an underlying differentially algebraic equation for the susceptibility, ruling out a number of possible differentially algebraic forms.
\end{abstract}

PACS: 05.50.+q, 05.10.-a, 02.30.Gp, 02.30.Hq, 02.30.Ik

AMS Classification scheme numbers: 03D05, 11Yxx, 33Cxx, 34Lxx, 34Mxx, 34M55, 39-04, 68Q70 
Key-words: non-holonomic functions, differentially algebraic functions, differentially transcendental functions, closure properties, non-linear differential equations, susceptibility of the Ising model, modulo prime calculations, algebraic functions, composition of functions, diagonals of rational functions, algebraic power series.

\section{Introduction}

The two-dimensional Ising model is arguably the most important statistical mechanical model ever conceived, historically, pedagogically, and in terms of its applications. Historically, the zeroth field-derivative of the free-energy was found by Onsager [1] in 1944. The internal energy, given by the temperature derivative of the free-energy, can be expressed in terms of integrals of complete elliptic integrals of the third kind, or ${ }_{4} F_{3}$ hypergeometric functions [2], and is therefore a $D$-finite or holonomic function. See Appendix A for a brief introduction to D-finite and D-algebraic functions, as well as some fundamental results about their behaviour.

The first field-derivative is the spontaneous magnetisation, and was publicly presented without proof by Onsager in 1949, and proved by Yang [3] in 1952. It is a simple algebraic function.

The second field-derivative is the susceptibility $\chi$, and no closed form expression is known. Despite this, we have a huge body of knowledge about the susceptibility. In $1976 \mathrm{Wu}, \mathrm{McCoy}$, Tracy and Barouch [4 showed that it could be expressed as an infinite sum of $n$-dimensional integrals, $\chi^{(n)}$, so that

$$
\chi=\sum_{n \text { even }} \chi^{(n)}, \quad \text { for } T<T_{c}, \quad \text { and } \quad \chi=\sum_{n \text { odd }} \chi^{(n)}, \quad \text { for } T>T_{c} .
$$

For the low-temperature susceptibility, only even powers of $n$ contribute, starting at $n=2$, while for the high-temperature susceptibility, only odd powers contribute. In 2001, Orrick et al. [5 proved that each of these integrals $\chi^{(n)}$ is D-finite, but argued that their infinite sum, that is, the full susceptibility $\chi$ itself, is not. In fact, this follows from earlier work of Nickel [6] who showed that the susceptibility has a natural boundary in the complex plane, and such functions cannot be D-finite.

In a series of papers, Maillard and co-workers 17, 8, 9, 10, 11, 12 found the linear ordinary differential equations (ODEs) satisfied by $\chi^{(3)}, \cdots, \chi^{(6)}$, and showed how, with sufficient computer power, others could, in principle, be found. $\chi^{(1)}$ is algebraic, and $\chi^{(2)}$ satisfies a low-order ODE, but the order of the linear ODEs satisfied by $\chi^{(n)}$ increases rapidly with $n$ as does the degree of the polynomial coefficients of each derivative, so the number of series coefficients needed to discover the linear ODE also increases rapidly with $n$. In 2011, Chan et al. 13] extended the work of Orrick et al. to other two-dimensional lattices, and gave an expansion of the scaled form of the susceptibility to unprecedented accuracy.

Accepting that the susceptibility is not D-finit $\$$, the question arises as to which class of functions the susceptibility belongs? In earlier work [15, based on a series expansion of 2000 terms we showed that modulo powers of 2, one cannot distinguish the full susceptibility from some simple diagonals of rational functions $\mathrm{S}$ that reduce

$\ddagger$ No rigorous proof of this result exists, but no reasonable person doubts it.

$\S$ These are known to be D-finite - see Appendix A 
to algebraic functions modulo $2^{r}$. However, for other primes we were unable to find corresponding algebraic function reductions.

From another perspective, one may ask which class of functions is the natural generalisation of D-finite functions, and then try to determine whether or not the Ising susceptibility belongs to that class. The answer, or at least one answer, is the class of differentially algebraic functions. A series $F(x)$ is called a differentially algebraic, or simply $D$-algebraic, if there exists a polynomial $P$ such that $F(x)$ satisfies a polynomial differential equation of finite order $k$ :

$$
P\left(x, F(x), F^{\prime}(x), \ldots, F^{(k)}(x)\right)=0 .
$$

where $F^{(k)}(x)$ denotes the $k$-th derivative. Note that a $D$-finite function can also be seen as the solution of differentially algebraic ODEs. A relevant example is the ODE for the diagonal correlations $C(N, N)$ of the square Ising model. While this satisfies a $D$-finite ODE, it is also known to satisfy a differentially algebraic equation, notablyt (a sigma-form of) Painlevé VI (see, for instance, equation (9) in [14]).

In this paper we investigate the possibility that the susceptibility is a differentially algebraic function. We tackle this from several perspectives. Firstly, we report on a dramatic extension of the susceptibility series from 2000 terms to 5043 terms. We use this extended data in two different ways. The first is to provide further confirmation of the results found earlier [15], that the susceptibility reduces to algebraic functions modulo $2^{r}$, and unfortunately to discover that even with these much longer series we were unable to find a similar algebraic function reduction modulo other primes. If the susceptibility were holonomic, or indeed differentially algebraic, then with a sufficient number of terms we could, in principle, find the underlying ODE (given appropriate levels of computer power and precision). Then we could predict the next 100, or 1000 or whatever coefficients. If these agreed with the exact coefficients, then there would be no doubt that the exact ODE had been found. While that would not be a rigorous proof, it would undoubtedly be correct, and form the basis from which a rigorous proof could be constructed.

The second approach is to use an algorithm, written by one of us [16], to search for differentially algebraic functions. With such a long series, we are able to rule out algebraic differential equations of quite high order. Finding the susceptibility in this way would be almost too good to be true, so while disappointing, this negative result is not totally unexpected.

In order to further explore the possibility that the susceptibility might be differentially algebraic, we have embarked on a study of the behaviour of such functions when their series expansions have integer coefficients, which is of course the case for the susceptibility. We study several examples of differentially algebraic functions whose series expansions have integer coefficients. For all the examples we choose, we find that the series reduce modulo $p^{r}$ to an algebraic function (henceforth, we use "modulo $p^{r}$ " to mean "modulo a prime or a power of a prime"). There are known counterexamples to this observation in the form of series with Hadamard gaps [17, 18, 19, such as the theta function $\theta_{3}(0, q)$. However, if their expansion variable is changed from the nome to the modulus, they are no longer counterexamples. This kind of lacunary series exception is not expected to be the kind of standard series we will encounter in lattice statistical mechanics.

$\dagger D$-finite functions can be expressed as solutions of (an infinite number of) differentially algebraic equations with movable singularities. However, that $D$-finite functions can also be solutions of differentially algebraic equations with fixed critical points is quite remarkable. 
We also wish to make a connection between our earlier observation that the susceptibility reduces to algebraic functions modulo $2^{r}$ and the behaviour we observe in the examples of differentially algebraic functions we study. We first note that functions expressible as diagonals of rational functions 20, see (Appendix A.3), must bE $\#$ D-finite, as proved by Lipshitz 21. Secondly, as proved by Furstenberg 22, the diagonal of a rational power series must be an algebraic series modulo $p$ for almost all primes $p$.

Our studies of miscellaneous examples of power series with integer coefficients, non-zero radius of convergence and no Hadamard gaps [17, 18, 19, that are differentially algebraic show that they reduce, modulo most $p^{r}$ to an algebraic function. This suggests that a large set of such series might be seen as a natural generalisation of diagonals of rational functions. We provide abundant evidence that this is a reasonable, and useful, working hypothesis.

Connecting our examples to the susceptibility problem, we observe that for many simple examples it often requires thousands, or even tens of thousands, of coefficients to find the corresponding algebraic equations modulo even a small prime. This is consistent with the behaviour we have found for the susceptibility, and leaves open the question whether the susceptibility is differentially algebraic.

A broader aim of this paper is to understand the class of non-holonomic power series with integer coefficients that reduce, modulo (almost every) $p^{r}$ to algebraic functions [23]. We showed [24] that a non-linear ODE, obtained by Tutte [25, 26] for the generating function of the $q$-coloured rooted triangulations by vertices, belongs to this class, at least for $q=4$.

Not all differentially algebraic power series with integer coefficients reduce to algebraic functions modulo almost every $p^{r}$. For instance, the generating function of the squares $\sum_{n \geq 1} x^{n^{2}}$ (which amounts to considering the theta function as a function of the nome $q$, namely $\left.\theta_{3}(0, q)=1+2 \sum_{n>1} q^{n^{2}}\right)$ is well-known to be differentially algebraic [27, 28, but does not reduce modulo $p^{r}$ to an algebraic function \$, as a consequence of Cobham's [29] theorem, which, in essence, states that if a series has only coefficients 0 or +1 it can be algebraic modulo two successive primes only if it is rational. We will, however, present a large accumulation of examples of differentially algebraic power series with integer coefficients (including divergent series) which reduce modulo primes to algebraic functions.

The results found for Tutte's generating function 24, with $q=4$, as well as the accumulated examples referred to above, naturally raise the question whether, or to what extent, "most" differentially algebraic power series with integer coefficients reduce modulo almost every $p^{r}$ to algebraic functions. This would then suggest that "most" differentially algebraic power series with integer coefficients can be seen as some sort of "non-linear" generalisation of diagonals of rational functions. Recall that diagonals of rational functions are holonomic. They are well-known to reduce to algebraic functions modulo $p^{r}$ [20. Note that differentially algebraic power series with integer coefficients are an obvious generalisation of D-finite series with integer coefficients, and such D-finite series appear to be algebraic modulo almost all $p^{r}$ (see

$\ddagger$ Conversely, there is a conjecture due to Christol [20] that a D-finite power series with integer coefficients and non-zero radius of convergence is the diagonal of a rational power series.

$\S$ The results are different if one views these functions as functions of the modulus $k$ instead of the nome $q$. For instance, the Eisenstein series $E_{4}$, when seen as a function of the modulus $k$, is holonomic, and can be expressed in terms of complete elliptic integrals of the first kind $K(k)$, and thus reduces to algebraic functions modulo primes and power of primes: $E_{4}(q)=K(k)^{4} \cdot\left(1-k^{2}\left(k^{\prime}\right)^{2}\right)$. 
Conjecture 12 and Section 5 in [30]). The difference between diagonals of rational functions and D-finite series with integer coefficients, corresponds to "Christol's conjecture", see 20, and for instance the ${ }_{3} F_{2}$ hypergeometric example in Section 5.1 ,

The class of differentially algebraic power series has a very important property: it is closed under the composition of function $\llbracket$. The class of non-holonomic power series with integer coefficients that reduce to algebraic functions modulo $p^{r}$ is probably also closed under the composition of functions.

A subset, the class of differentially algebraic power series with integer coefficients that reduce to algebraic functions modulo $p^{r}$ is an extremely large class. We will see in this paper that 5043 coefficients for the susceptibility series of the square-lattice Ising model is still insufficient to see if the square-lattice Ising susceptibility series reduces to an algebraic function modulo $p^{r}$ for $p \neq 2$. This suggests that trying to see if the Ising susceptibility series is a differentially algebraic power series might be a simpler question to address.

\section{Revisiting Tutte's differentially algebraic generating function.}

Before trying to find new results on the square-lattice Ising susceptibility, we will revisit 24] a simpler (but already important and non-trivial) example of a non-holonomic function namely Tutte's generating function for $q$-coloured rooted triangulations by vertices [25, 26],

$$
H(x)=q \cdot(q-1) \cdot x^{2}+q \cdot(q-1) \cdot(q-2) \cdot \sum_{n=3}^{\infty} P_{n}(q) \cdot x^{n},
$$

considered from the perspective of its reducability modulo $p^{r}$. The coefficients of the series (3) can be obtained from a simple quadratic recurrence relation:

$$
\begin{gathered}
q \cdot(n+1) \cdot(n+2) \cdot h_{n+2}=q \cdot(q-4) \cdot(3 n-1) \cdot(3 n-2) \cdot h_{n+1} \\
+2 \sum_{i=1}^{n} i \cdot(i+1) \cdot(3 n-3 i+1) \cdot h_{i+1} h_{n-i+2},
\end{gathered}
$$

that can also be rewritten as

$$
\begin{gathered}
q \cdot(q-4) \cdot\left(9 n(n+1) \cdot h_{n+1}-18(n+1) \cdot h_{n+1}+20 \cdot h_{n+1}\right) \\
+2 \sum_{i=1}^{n}\left(i \cdot(i+1) \cdot h_{i+1}\right) \cdot\left(6 \cdot(n-i+2) \cdot h_{n-i+2}-10 \cdot h_{n-i+2}\right) \\
\quad-q \cdot(n+1) \cdot(n+2) \cdot h_{n+2}=0 .
\end{gathered}
$$

The generating function (3) is a solution of the differentially algebraic equation [24]:

$$
\begin{aligned}
q \cdot( & q-4) \cdot\left(9 x^{2} \frac{d^{2} H(x)}{d x^{2}}-18 x \frac{d H(x)}{d x}+20 H(x)\right) \\
& +\left(6 x \frac{d H(x)}{d x}-10 H(x)-q x\right) \cdot \frac{d^{2} H(x)}{d x^{2}}=2 q^{2} \cdot(1-q) \cdot x .
\end{aligned}
$$

|| The composition of two differentially algebraic functions is differentially algebraic, see Remark 4.3 in Lipshitz and Rubel 32 and, in the particular case of constructible differentially algebraic series, Bergeron and Reutenauer [33. We thank D. Bertrand for providing a proof of Ehud Hrushovski of that result, in the most general framework. Note that differentially algebraic functions are not closed under Hadamard product, Laplace transform, or inverse Laplace transform [32]. 
It is quite easy to deduce (6) from (5), the rhs of (6) depending on the initial conditions, namely the first terms of the series (3).

In 24] we have shown that, in the $q=4$ case, this generating function

$$
\begin{aligned}
H(x)= & 12 x^{2}+24 x^{3}+168 x^{4}+1656 x^{5}+19296 x^{6}+248832 x^{7} \\
& +3437424 x^{8}+49923288 x^{9}+753269856 x^{10}+\cdots
\end{aligned}
$$

is non-holonomic and reduces to an algebraic function modulo every prime or power of a prime. Let us consider other solutions of (6) for $q=4$, corresponding to different initial condition $\forall$ namely the one-parameter family of solutions (here $A$ denotes that parameter):

$$
H_{A}(x)=(A-1) \cdot x+12 \frac{x^{2}}{A}+24 \frac{x^{3}}{A^{3}}+168 \frac{x^{4}}{A^{5}}+1656 \frac{x^{5}}{A^{7}}+\cdots
$$

This one-parameter family of solutions can be written

$$
H_{A}(x)=-x+A^{3} \cdot\left(\frac{x}{A^{2}}+H\left(\frac{x}{A^{2}}\right)\right),
$$

where the function $H(x)$ in (9) is the series (7). Since (77) reduces to algebraic functions modulo each prime or power of a prime, one sees immediately that these other solutions (8) of the non-linear differential equation (6) for $q=4$, also reduce to algebraic functions modulo every prime or power of a prime.

\subsection{Tutte's differentially algebraic generating function for other values of $q$.}

It is tempting to see if one also has reduction to algebraic functions modulo $p^{r}$ for values of $q$ other than $q=4$. Tutte's algebraic differential equation (6) is known [34, 35] to provide algebraic solutions for all the Tutte-Beraha numbers, and in fact, for all $q$ of the form $q=2+2 \cos (j \pi / m)$ ( $j$ and $m$ are integers). Therefore we have considered Tutte's differentially algebraic equation (6) for miscellaneous values that are not of the form $q=2+2 \cos (j \pi / m)$, for instance integer values larger than $4, q=5,7, \ldots$, or rational values $q=M / N$.

For instance, for $q=5$, one finds that the series (3) still reduces to algebraic functions modulo every prime. Modulo 7 the series (3) for $q=5$, namely $6 x^{2}+4 x^{3}$ $+2 x^{4}+3 x^{5}+5 x^{6}+6 x^{7}+5 x^{8}+5 x^{9}+\cdots$, is a solution of the polynomial equation

$$
\begin{aligned}
& F(x)^{5}+\left(5 x^{2}+6 x+6\right) \cdot F(x)^{4}+\left(x^{4}+2 x^{3}+2 x^{2}+4 x+4\right) \cdot F(x)^{3} \\
& \quad+x^{2} \cdot\left(6 x^{2}+6 x+1\right) \cdot F(x)^{2}+x^{2} \cdot\left(2 x^{5}+5 x^{3}+5 x^{2}+x+5\right) \cdot F(x) \\
& \quad+x^{4} \cdot\left(2 x^{5}+4 x^{4}+3 x^{3}+x^{2}+2 x+5\right)=0 .
\end{aligned}
$$

Modulo 11 the $q=5$ series, namely $9 x^{2}+5 x^{3}+x^{5}+7 x^{6}+9 x^{7}+10 x^{8}+8 x^{9}+\cdots$, is a solution of the polynomial equation

$$
F(x)^{3}+7 \cdot F(x)^{2}+x \cdot\left(4 x^{2}+7 x+9\right) \cdot F(x)+x^{3} \cdot\left(2+3 x+10 x^{2}\right)=0,
$$

and modulo 13 the $q=5$ series is a solution of a polynomial equation of degree 15 in $F(x)$, and degree 27 in $x$. Similarly to $q=4$ (see (8)), the other solutions of (6) for $q=5$ also reduce to algebraic functions modulo every prime, or power of a prime.

We give in Appendix B many other examples of reductions modulo primes of the solutions of (6) for various values of $q$ that are not of the form $q=2+2 \cos (j \pi / m)$.

$\dagger$ Note a missprint in [24]: one should read " $h_{0}=0$ but does not impose $h_{1}=0$ " instead of " $h_{0}=0$ and $h_{1}=0$, but does not impose $h_{2}=q(q-1)=12$ ". 
Tutte's differentially algebraic equation (6) provides a non-trivial, important but simple enough, "toy example" of reduction modulo primes or powers of primes, of differentially algebraic functions. We also give in Appendix C an example of a (quite natural) generalisation of Tutte's differentially algebraic equation (6) that also yields power series solutions with integer coefficients reducing to algebraic functions modulo $p^{r}$.

We have accumulated similar results for large sets of solutions of miscellaneous differentially algebraic functions having power series solutions with integer coefficients, in particular power series generated by simple quadratic recursions (similar to the one generating the solution series (3), (see equation (5) in [24])). Such examples are displayed in Appendix D. One sees from these examples that, even if these differentially algebraic power series are divergent series, they still reduce to algebraic functions modulo primes or powers of primes.

These exploratory results on miscellaneous non-linear equations suggest that differentially algebraic power series solutions with integer coefficients and no Hadamard gaps usually reduce to algebraic functions modulo primes, or powers of primes. If that is the case, recalling that diagonals of rational functions are wellknown to reduce to algebraic functions modulo $p^{r}$ [20, then differentially algebraic power series could be seen as a natural "non-linear" generalisation of diagonals of rational functions.

\section{Brain storming: square-lattice Ising susceptibility and diagonals of rational functions.}

One of the simplest examples of a differentially algebraic equation is the Chazy III equation. It is worth considering as a toy example of some properties of the Ising susceptibility because it has the Painlevé property expressed as ratios of holonomic functions (see Appendix A.3). Recall that the ratio (not the product!) of two holonomic functions is generically non-holonomic. The ratio $\tau(x)=y_{1} / y_{2}$ of two solutions of the linear ODE, $\frac{d^{2} y}{d x^{2}}+R(x) \cdot y=0$, is a solution of the Schwarzian equation $\{\tau(x), x\}=2 R(x)$. Considering ratios of holonomic functions is thus a very simple way to build (selected) non-holonomic examples. In fact, these solutions can be expressed as a ratio of diagonals of rational functions, which implies that they have (circular) natural boundaries 37, 38, which is also observed to be a property of the susceptibility of the Ising model $[9]$.

The Chazy III equation [37, 38 is a third-order non-linear differential equation the solutions of which have a natural boundary [28]. It is

$$
\frac{d^{3} y}{d x^{3}}=2 y \frac{d^{2} y}{d x^{2}}-3\left(\frac{d y}{d x}\right)^{2},
$$

which can be rewritten in terms of a Schwarzian derivative [39, 40,

$$
f^{(4)}=2 f^{\prime 2} \cdot\{f, x\}=2 f^{\prime} f^{\prime \prime \prime}-3 f^{\prime \prime 2} \quad \text { with } \quad y=\frac{d f}{d x},
$$

where $\{\cdot, \cdot\}$ denotes the Schwarzian derivative.

$\ddagger$ There is little need to emphasise the importance of the Painlevé property to the square-lattice Ising model: the diagonal correlation functions are known [14, 36] to be solutions of (a $\sigma$ form of) Painlevé VI. 
In 24] it has been remarked that ratios of particular holonomic functions, namely ratios of diagonals of rational functions, automatically reduce to algebraic functions modulo every prime, or power of a prime, which is also a property we observed for the susceptibility series modulo powers of 2 .

The non-holonomic susceptibility series is known to be an infinite sum of holonomic functions [9, 4, namely the $n$-fold integrals [7, 8, 10, 11, 12, 41] $\chi^{(n)}$ that are themselves diagonals of rational functions 20, 29, 42, 43, 44. To some extent, the remarkable result [15] that the non-holonomic susceptibility series reduces to algebraic functions modulo $2^{r}$ can be seen as a property of these diagonal of rational functions, namely that these $\chi^{(n)}$ reduce to zero modulo $2^{r}$ when $n$ is large enough [24.

All these ideas suggest that it may be useful to take a fresh look at the squarelattice Ising susceptibility from the perspective of a "diagonals of rational function approach" 20. The "Chazy III scenario" just discussed, suggesting that the squarelattice Ising susceptibility might just be a ratio of diagonals of rational functions reducing to algebraic functions modulo primes, and, possibly the occurrence of natural boundaries, is certainly far too naive. If that were the case, then the Ising susceptibility would easily be seen to reduce to algebraic functions not only modulo $2^{r}$, but also modulo any power of a prime, which does not seem to be the case [15]. Accordingly we explore more involved scenarios.

\section{More involved constructions from diagonals of rational functions.}

If ratios of diagonals of rational functions are automatically such that they reduce to algebraic functions modulo $p^{r}$ and are also differentially algebraic functions (see Section 5.2 and Appendix D in [24]), one can easily imagine more involved expressions of diagonals of rational functions that will also reduce to algebraic functions modulo $p^{r}$ 24]. Note that the class of rational expressions of diagonals of rational functions reduces to the previous class of ratios of diagonals of rational functions. This is a consequence of the fact that polynomial expressions of diagonals of rational functions are themselves diagonals of rational functions. To get a larger class, one needs to consider, at least, algebraic functions of diagonals of rational functions (as already suggested in 24]). We look at some of these in the next subsection.

\subsection{Algebraic functions of diagonals of rational functions.}

Algebraic functions of diagonals of rational functions, which are generically nonholonomic, reduce to algebraic functions modulo $p^{r}$ and are also differentially algebraic.

Let us just give a simple heuristic and pedagogical example. Consider two ${ }_{2} F_{1}$ hypergeometric functions which are also diagonals of rational functions

$$
H_{1}={ }_{2} F_{1}\left(\left[\frac{1}{2}, \frac{1}{2}\right],[1], 16 \cdot 20 \cdot x^{2}\right), \quad H_{2}={ }_{2} F_{1}\left(\left[\frac{1}{3}, \frac{1}{3}\right],[1], 27 \cdot 20 \cdot x^{2}\right),
$$

and consider the two roots of

$$
z^{2}-2 H_{1} \cdot z+H_{2}=0
$$


namely $z_{ \pm}=H_{1} \pm\left(H_{1}^{2}-H_{2}\right)^{1 / 2}$, which have series expansions with integer coefficients

$$
\begin{aligned}
z_{-}(x) & =1-10 x+80 x^{2}-1040 x^{3}+14400 x^{4}-145920 x^{5}+3200000 x^{6} \\
& -10992320 x^{7}+784000000 x^{8}+6780473600 x^{9}+203212800000 x^{10} \\
& +5987941079040 x^{11}+54641664000000 x^{12}+3543158723957760 x^{13} \\
& +15076638720000000 x^{14}+\cdots
\end{aligned}
$$

and $z_{+}(x)=z_{-}(-x)$. Apart from the coefficients of $x, x^{3}, x^{5}, x^{7}$, all the coefficients are positive integers.

These two series are not holonomic (square roots of holonomic functions are generically not holonomic), rather they are differentially algebraic. The expression $H_{1}^{2}-H_{2}$ in (13) is holonomic (it is a simple polynomial of holonomic functions), being the solution of a linear differential operator $L_{5}$ of order five. The square root of that holonomic function $g(x)$, defined by $g(x)^{2}=H_{1}^{2}-H_{2}$, is differentially algebraic, and is the solution of the polynomial differential equation $L_{5}\left(g(x)^{2}\right)=0$. Straightforward (but tedious) differential algebra calculations show that the series (14) is the solution of the order-seven polynomial differential equation

$$
P\left(x, F(x), F^{\prime}(x), F^{\prime \prime}(x), F^{\prime \prime \prime}(x), F^{(4)}(x), F^{(5)}(x), F^{(6)}(x), F^{(7)}(x)\right)=0,
$$

where $F(x)$ denotes the series (14), and where $F^{(n)}(x)$ denotes its $n^{\text {th }}$ derivative. The polynomial $P$ is the sum of 3769 monomials of degree at most 46 in $x, 4$ in $F(x)$, $F^{\prime}(x), F^{\prime \prime}(x)$, and $F^{\prime \prime \prime}(x), 3$ in $F^{(4)}(x)$ and $F^{(5)}(x), 2$ in $F^{(6)}(x)$, and 1 in $F^{(7)}(x)$. The complexity of this polynomial should be compared with the simplicity of the original function (13).

The polynomial equation (15) can be written as:

$$
\sum_{n=0}^{46} x^{n} \cdot P_{n}\left(F(x), F^{\prime}(x), \cdots, F^{(7)}(x)\right)=0,
$$

where the polynomials $P_{n}$ are homogeneous polynomials of degree 11 in $F(x), F^{\prime}(x), \cdots F^{(7)}(x)$. If one scales $F(x)$ as $F(x) \rightarrow A \cdot F(x)$, (and consequently $\left.F^{(i)}(x) \rightarrow A \cdot F^{(i)}(x)\right)$, the polynomial $P$ in (15) scales as $P \rightarrow A^{4} \cdot P$. Therefore, if $F(x)$ is a solution of (15), $A \cdot F(x)$ is also a solution of (15).

It is straightforward to see that the non-holonomic series (14) reduces to an algebraic function modulo $p^{r}$ (see Appendix E).

The class of algebraic functions of diagonals of rational functions is already a very large one. However, we will see in the next section (4.2) that power series with integer coefficients reducing to algebraic functions modulo every prime, or power of a prime, corresponds to a much larger class.

\subsection{Compositions of diagonals of rational functions.}

Let us consider another simple example corresponding to the composition of two holonomic functions (in fact, the composition of two diagonals of rational functions).

Before introducing that example, recall the closure properties of holonomic functions. They are closed under the operations of addition, multiplication, indefinite integration, differentiation and right composition with algebraic functions. In other words if $H(x)$ denotes a holonomic function, and $A(x)$ an algebraic function, $H(A(x))$ is necessarily holonomic. In constrast, if one performs the composition of two holonomic (but non-algebraic) functions, the result is generically not holonomic. For 
instance, the reciprocal function $R(x)=1 / x$ is holonomic, but the composition of $R(x)$ with a holonomic function $H(x)$, namely $R(H(x))=1 / H(x)$ is generically nonholonomic. These closure properties extend to diagonals of rational functions. For instance, the class of diagonals of rational functions is also closed under the operations of addition, subtraction, multiplication, indefinite integration, differentiation and right composition with algebraic functions.

Consider the two holonomic functions (that are diagonals of rational functions [20])

$$
H_{1}(x)={ }_{2} F_{1}\left(\left[\frac{1}{2}, \frac{1}{2}\right],[1], 16 x\right), \quad H_{2}(x)=x H_{1}(x),
$$

and consider the following composition of these two holonomic functions:

$$
H_{1}\left(H_{2}(x)\right)={ }_{2} F_{1}\left(\left[\frac{1}{2}, \frac{1}{2}\right],[1], 16 x \cdot{ }_{2} F_{1}\left(\left[\frac{1}{2}, \frac{1}{2}\right],[1], 16 x\right)\right) .
$$

This function has the following series expansion with integer coefficients:

$$
\begin{aligned}
& H_{1}\left(H_{2}(x)\right)=1+4 x+52 x^{2}+832 x^{3}+14468 x^{4}+263072 x^{5}+4919728 x^{6} \\
& \quad+93824000 x^{7}+1815689828 x^{8}+35542852576 x^{9}+702276985968 x^{10} \\
& \quad+13984093836288 x^{11}+280299095853776 x^{12}+5650349273844992 x^{13} \\
& \quad+114466793551793216 x^{14}+2329040212651647488 x^{15}+\cdots
\end{aligned}
$$

The coefficients of this series grow like $\lambda^{n}$ where $\lambda \simeq 21.7257468152791$. The radius of convergence of this series $1 / \lambda$, is $x_{c} \simeq 0.046028337184$, which is expected to be a transcendental values such that:

$$
16 x_{c} \cdot{ }_{2} F_{1}\left(\left[\frac{1}{2}, \frac{1}{2}\right],[1], 16 x_{c}\right)=1 .
$$

It is reassuring that, despite the fact that the series (19) is not holonomic, nevertheless a (linear) differential Padé analysis on 1654 terms of the series (19) gives a dominant singularity at 0.046028337184 (in complete agreement with (20)) and, furthermore, gives an exponent equal to 0 corresponding to a logarithmic singularity. One also sees a number of singularities around 1/16, characteristic of an irregular singularity, see Appendix I.

Modulo 5 the series (19) reads:

$$
\begin{aligned}
& H_{1}\left(H_{2}(x)\right)=1+4 x+2 x^{2}+2 x^{3}+3 x^{4}+2 x^{5}+3 x^{6}+3 x^{8}+x^{9}+3 x^{10} \\
& \quad+3 x^{11}+x^{12}+2 x^{13}+x^{14}+3 x^{15}+3 x^{16}+2 x^{17}+4 x^{19}+2 x^{20}+x^{22} \\
& \quad+x^{23}+4 x^{24}+x^{25}+3 x^{26}+\cdots
\end{aligned}
$$

The previous holonomic function $H_{2}(x)$ (which is also a diagonal of a rational function [20]) reduces, modulo 5 , to a very simple algebraic function $A_{2}(x)$ :

$$
\begin{aligned}
& H_{2}(x) \bmod 5=A_{2}(x)=\frac{x}{\left(1+4 x+x^{2}\right)^{1 / 4}}=x+4 x^{2}+x^{3}+4 x^{6}+x^{7} \\
& +4 x^{8}+x^{11}+4 x^{12}+x^{13}+4 x^{26}+x^{27}+4 x^{28}+x^{31}+4 x^{32}+\cdots \quad(22)
\end{aligned}
$$

Naively one might expect, modulo 5 , the series (19) for $H_{1}\left(H_{2}(x)\right)$, namely (21) to also be the same as the series expansion of $H_{1}\left(A_{2}(x)\right)$ modulo 5. Indeed, if one

$\S$ For a transcendental condition like 20], one might expect, at first sight, an infinite number of (complex) transcendental values for $x_{c}$. This is not the case here. 
performs the series expansion of $H_{1}\left(A_{2}(x)\right)$ modulo 5 , one gets

$$
\begin{aligned}
& H_{1}\left(A_{2}(x)\right)={ }_{2} F_{1}\left(\left[\frac{1}{2}, \frac{1}{2}\right],[1], 16 \frac{x}{\left(1+4 x+x^{2}\right)^{1 / 4}}\right) \\
& =1+4 x+2 x^{2}+2 x^{3}+3 x^{4}+2 x^{5}+3 x^{6}+3 x^{8}+x^{9}+3 x^{10}+3 x^{11} \\
& +x^{12}+2 x^{13}+x^{14}+3 x^{15}+3 x^{16}+2 x^{17}+4 x^{19}+2 x^{20}+x^{22}+x^{23} \\
& +4 x^{24}+x^{25}+3 x^{26}+\cdots
\end{aligned}
$$

which is (as expected) the same expansion as (21). However, recalling the previously mentioned closure properties of diagonals of rational functions by right composition with algebraic functions, the form $H_{1}\left(A_{2}(x)\right)$ in (23) can also be viewed as a diagonal of a rational function and, thus, the series (24) should be the expansion of an algebraic function. Not surprisingly it is also the series expansion of $A_{1}\left(A_{2}(x)\right)$ modulo 5 .

We found the algebraic function satisfying, modulo 5 , the polynomial equation:

$$
\begin{aligned}
\left(x^{8}\right. & \left.+x^{6}+4 x^{5}+2 x^{4}+3 x^{3}+3 x^{2}+3 x+1\right) \cdot F(x)^{16} \\
& +\left(x^{4}+3 x^{3}+3 x^{2}+3 x+1\right) \cdot\left(F(x)^{12}+F(x)^{4}+1\right) \\
& +\left(x^{2}-x+1\right) \cdot\left(3 x^{4}+5 x^{3}+6 x^{2}+4 x+1\right) \cdot F(x)^{8}=0 .
\end{aligned}
$$

Of course there is nothing special about the prime 5: the series clearly reduces to algebraic functions modulo primes, or powers of a prime (see Appendix F.

The other solutions of the differentially algebraic equation (15) also reduce to algebraic functions modulo primes, or powers of a prime (see Appendix H.2.

Such simple results provide a strong incentive to systematically study the reduction, modulo primes, or powers of a prime, of all the solution-series with integer coefficients of differentially algebraic equations, and more generally all globally bounded Appendix A.4 20]) solution-series of differentially algebraic equations.

\subsection{Composition of holonomic functions are differentially algebraic.}

Recalling the previous composition of two simple ${ }_{2} F_{1}$ hypergeometric functions that are diagonals of rational functions, namely (18), it is straightforward (but quite tedious), to find that the composition (18) of these two holonomic functions is actually differentially algebraic, and, more generally, that any composition of holonomic functions is differentially algebraic. This is a particular case of the more general result that the composition of two differentially algebraic functions is differentially algebraic (see [33]). In this example, denoting the series (18) by $F(x)=H_{1}\left(H_{2}(x)\right.$ ), one finds that it satisfies a polynomial equation

$$
P\left(x, F(x), F^{\prime}(x), F^{\prime \prime}(x), F^{\prime \prime \prime}(x), F^{(4)}(x)\right)=0,
$$

where the polynomial $P$ is the sum of 9972 monomials of degree at most, 32 in $x$, 6 in $F(x), 11$ in $F^{\prime}(x), 11$ in $F^{\prime \prime}(x), 7$ in $F^{\prime \prime \prime}(x)$ and 4 in $F^{(4)}(x)$. This polynomial equation can be written as

$$
\sum_{n=0}^{32} x^{n} \cdot P_{n}\left(F(x), F^{\prime}(x), F^{\prime \prime}(x), F^{\prime \prime \prime}(x), F^{(4)}(x)\right)=0,
$$

where the polynomials $P_{n}$ are homogeneous polynomials of degree 11 in $F(x), F^{\prime}(x), \cdots, F^{(4)}(x)$. If one scales $F(x)$ as $F(x) \rightarrow A \cdot F(x)$, (and consequently $\left.F^{(i)}(x) \rightarrow A \cdot F^{(i)}(x)\right)$, the polynomial $P$ in (26) scales as $P \rightarrow A^{11} \cdot P$. Therefore, if $F(x)$ is a solution of (26), $A \cdot F(x)$ is also a solution of (26). 
This is a general result: for compositions of holonomic functions, the differentially algebraic equation inherits the (differential Galois) symmetries of the linear differential operator corresponding to the first holonomic function in the composition (see Appendix G.

Remark 1: The non-linear differential equation (26) has, of course, many more solutions (see Appendix H.1) than the solution given by the series (19) for which one has a very precise location for one critical point namely the transcendental value $x_{c} \simeq 0.04602833718455$, see (20). It is clear that, in contrast with linear differential equations, where the (algebraic) critical points correspond to the zeros of the head polynomial of the linear differential operator, such a (transcendental) critical value (20) cannot be simply seen in the non-linear ODE (27). The singularities of the solutions of (27) depend on the "initial conditions", the first terms of the solution series: these singularities are movable singularities (see Appendix H.1).

The occurence of movable singularities (that can be infinite in number ...) is a phenomenon that we will see occur systematically in almost all the differentially algebraic equations emerging in our studies.

Along this line it is interesting to remark that the traditional diff-Padé analysis performed on so many series of lattice statistical mechanics or enumerative combinatorics, tries to associate to a given series the best linear differential equation with polynomial coefficients, which necessarily has fixed singularities. With this differentially algebraic example (18), which requires a non-linear differential equation (27) with movable singularities to be correctly described, it is interesting to see the kind of results and bias that are obtained from the traditional diff-Padé analysis forcing, by construction, fixed singularities, on this movable singularities example. This is sketched in Appendix I

With the "toy example" (18), where one has a very precise location for one critical point, it is interesting to see what kind of results a traditional linear differential Padé analysis would give on such a non-linear differentially algebraic series (18). In a composition of two holonomic functions $H_{1}\left(H_{2}(x)\right)$, one might expect, in general, that the critical points that are not algebraic numbers anymore 20, but transcendental numbers, such that $H_{2}(x)$ is a singular point of $H_{1}(x)$, are, nevertheless all regular singular points, their critical exponents being simply related to the ones of the ${ }_{2} F_{1}$ hypergeometric series. The singular points of $H_{2}(x)$ may yield irregular singularities for $H_{1}\left(H_{2}(x)\right)$.

\subsection{Compositions of functions.}

From these simple examples we see that the composition of diagonals of rational functions, or even algebraic functions of diagonals of rational functions $\mathbb{\prod}$, build an extremely large class of functions: their series are globally bounded and, so, can be recast to have integer coefficients, a non-zero radius of convergence, and will reduce to algebraic functions modulo every prime and power of a prime, these functions being necessarily differentially algebraic.

One probably has a much more general result: if two series with integer coefficients are such that they reduce to algebraic functions modulo every $p^{r}$, so does the

\| In fact there is no reason to restrict ourselves to the composition of holonomic functions, like diagonals of rational functions. One can also compose algebraic functions of diagonals of rational functions (see 4.17). 
composition of these two serie $₫$. Note however that this class has no reason to be differentially algebraic. Actually, if two series $S_{1}$ and $S_{2}$ with integer coefficients (not necessarily differentially algebraic) reduce to algebraic functions $A_{1}$ and $A_{2}$ modulo every prime, or power of a prime, the composition $S_{1}\left(S_{2}(x)\right)$ reduces to $A_{1}\left(A_{2}(x)\right)$ modulo that prime, or power of a prime.

It is natural to ask if the class of arbitrary compositions of algebraic functions of diagonals of rational functions is sufficiently large to exhaust all series with integer coefficients, with non-zero radius of convergence, reducing modulo every prime (or power of a prime) to an algebraic function? This is, and this will probably remain for some time, an open question.

For such a large class of functions one can imagine that the reduction to algebraic functions modulo a prime can be difficult to see, the polynomial encoding this algebraic function being of very high degree (see Appendix E and Appendix Fin a differentially algebraic framework). Is this perhaps the situation we encounter, modulo 3,5 , etc. for the full susceptibility of the Ising model?

Since this very large class of functions obtained by compositions of an arbitrary number of diagonals of rational functions, or more generally, algebraic functions of diagonals of rational functions, actually corresponds to differentially algebraic functions, we have some incentive to see if the full susceptibility of the square-lattice Ising model is a differentially algebraic function.

\section{Getting 5043 terms for the susceptibility series generation.}

In earlier work [13, we described the generation and analysis of the Ising susceptibility series to 2048 term: $\boxplus$ on the square lattice in natural high- and low-temperature variables. By making some minor improvements to the program, and running for longer on a larger machine, we have extended the square-lattice series to 5043 terms 45.

These very long series confirm all the reductions to algebraic functions modulo $2^{r}$ obtained in [15. These new data would also allow the precision of the earlier analysis to be greatly enhanced. However we consider that there is little benefit in doing so, as the existing precision is so far in advance of any theoretical or experimental application that there is nothing to be gained in pursuing this (local) scaling approach.

As discussed in [5] and [6], the square-lattice Ising susceptibility is believed to have a natural boundary on the unit circle in the complex $s / 2=\sinh 2 K / 2$ plane and thus cannot be holonomic. Even with very long series it is extremely difficult to see this fundamental natural boundary property. Seizing the opportunity of the polynomialtime complexity of the algorithm [5], the motivation to get even longer series for the Ising susceptibility cannot be justified by a desire to extend such a (local) scaling analysis, or even an attempt to better understand the complex analytical structure of the susceptibility. In contrast the results obtained in [15] corresponding to the reduction modulo $2^{r}$ of the susceptibility series to algebraic functions, are of a more arithmetic and global character, and fully justify efforts to get series expansions with more than 2048 terms. Rather, we seek to investigate the (global) nature of the

ๆ If the two series also have a non-zero radius of convergence so does their composition.

+ Similar large series were also obtained and analysed with a totally different corner transfer matrix approach for the triangular and honeycomb lattices but only to 600 terms [13]. The singular part of the susceptibility was, however, obtained to great precision [13. 
susceptibility, as revealed by the behaviour of the coefficients modulo a prime, or power of a prime [15.

The program, which makes use of Fast Fourier Transform methods for multiplications, can be sped up essentially linearly with the number of processors. The series were calculated modulo many different primes of the form $p_{k}=2^{15}-r_{k}$ and the exact integer coefficients were then obtained from the set of remainders using the Chinese remainder procedure. The series coefficients $c_{n}$ grow asymptotically as $4^{n}$ so to get a series with $N$ terms requires approximately $2 N / 15$ primes. The calculation took some 22.5 hours per prime*. In order to obtain 5043 terms [45] of the (low and high-temperature) series we used 700 primes and the total calculation therefore required close to 16000 hours in total. This has been achieved using 700 processors of the National Computational Infrastructure (NCI) facility at the Australian National University. The parallel version of this program just runs several primes simultaneously.

Remark 1: It is tempting to imagine further extending these series, restricted modulo particular integers, for instance powers of 2 , to further check the reduction of the susceptibility series to algebraic functions modulo $2^{r}$. The current version the program performs integer divisions. This is only possible when one performs the calculation modulo a prime number. Therefore one cannot calculate modulo powers of 2 directly: one needs to calculate the series exactly (in characteristic zero) as has been done to 5043 terms and then from the exact series coefficients one can of course do any modulo integer calculations one wishes. Unless one can re-write the algorithm to avoid integer division there is little prospect of pushing these series modulo powers of a prime much further.

Remark 2: The nature of the algorithm unfortunately is such that one cannot run it modulo a small prime (like $3,5, \ldots$ ). The prime must be larger than the length of the series, due to the integer divisions that take place. In principle, one could calculate 10000 terms modulo a single prime greater than 10000. Unfortunately, when non-holonomic series reduce to algebraic functions modulo a prime $p$, we have seen 24 . that the complexity of the corresponding polynomial (encoding the algebraic function) grows with the prime $p$. The polynomials corresponding to these very long 10000 terms series would be huge, the 10000 terms being insufficient to find the polynomial (if they exist).

Remark 3: To get longer series would require one to perform the Chinese reminder procedure with more primes (each prime being larger than the length of the series). If we want to get 10000 terms for the susceptibility series, we would need to perform the calculations modulo more than 1350 different primes of the form $2^{15}-r_{k}$. Naturally if one used primes of the form say $2^{30}-r_{k}$ one would need half as many primes. However, in that case one would need to do integer multiplications using 64-bit integers. Furthermore the FFT multiplication of series is done using double-precision floating-point numbers (the remainders of the integer coefficients modulo the prime recovered from the floating-point numbers). The use of larger primes may require the use of higher precision floating-point calculations (such as quad-precision) in order to correctly reproduce the integer valued remainders. We note that a calculation of the 10000 term series took almost 68 hours (on a Mac Pro desktop with a $3.5 \mathrm{GHz} 6$-Core Intel Xeon E5 processor). So to get the exact series to order 10000 would require at

* On a newer machine the calculation per prime takes 12 hours, the 5043 terms calculation taking close to 6000 hours in total. 
least 90000 CPU hours on this machine.

\subsection{The 5043 terms series modulo $3,5,7, \ldots$ : no reduction to algebraic functions?}

Now that we have such long series for the susceptibility, we can revisit the results given in [15], namely that the (low or high temperature) susceptibility series reduce to algebraic functions modulo $2^{r}$. This is verified immediately. For instance, we find with 5043 terms of the high-temperature full susceptibility series $\tilde{\chi}_{H}(w)$, the simple functional equation modulo 32 (see equation (47) in [15):

$$
\tilde{\chi}_{H}\left(w^{2}\right)=w \cdot \tilde{\chi}_{H}(w)+8 w^{3} \cdot\left(2 w^{15}-w^{7}+w-5\right) .
$$

Modulo 64, 128, one verifies [15] with 5043 terms that one cannot distinguish between $\tilde{\chi}_{H}$ and $\tilde{\chi}_{H}^{(1)}+\tilde{\chi}_{H}^{(3)}+\tilde{\chi}_{H}^{(5)}$, which is the diagonal of a rational function [20, and thus reduces to an algebraic function modulo any prime or power of a prime, and in particular modulo 64, 128. Similarly, with the 5043 terms of the lowtemperature series of the full susceptibility $\tilde{\chi}_{L}$, one verifies [15] that one cannot, modulo $2,4,8,16,32,64,128,256$, distinguish between $\tilde{\chi}_{L}$ and $\tilde{\chi}_{L}^{(2)}+\tilde{\chi}_{L}^{(4)}+\tilde{\chi}_{L}^{(6)}$ which is the diagonal of a rational function [20, and thus reduces, modulo 2, 4, 8, 16, $32,64,128,256$, to an algebraic function. All the exact results of [15] remain valid with 5043 terms.

In contrast, the 5043 terms susceptibility series does not seem to reduce to algebraic functions even modulo $3,5,7,11$, etc. This is quite a puzzling result, when one compares this negative result with the comparatively easy way [15] reduction to algebraic functions are obtained modulo a large set of powers of 2 , and also the reasonably easy way 24] reductions to algebraic functions are obtained for the solutions of Tutte's equation (6), and the miscellaneous examples discussed in Appendix D.

At this stage it is not possible to conclude that this negative result with 5043 terms really means that the susceptibility series does not reduce to algebraic functions modulo $p^{r}$ for $p \neq 2$. It could be that the algebraic functions modulo $3,5,7,11$, etc are drastically more complicated, so that even a 5043 term series is too short.

With the simple quadratic example (13) of Section (4.1) we showed that this algebraic function of the diagonal of a rational function reduces modulo $p^{r}$ to an algebraic function. However, even in this very simple example, the corresponding polynomial is already, modulo 7, of degree 60 in $x$ and 72 in $z$ (see Appendix E. Switching from ratios of diagonals of rational functions (see Paragraph 5.2 of [24]) to algebraic functions of diagonals of rational functions may result in drastically more involved algebraic functions for the reductions modulo primes.

We found similar results for the composition of very simple holonomic functions of Section (4.2), and their reduction modulo 7 (which gave a polynomial equation of degree 36 in $z$ and 18 in $x$, see (F.3) in Appendix F). These two examples (algebraic expressions of diagonals of rational functions and compositions of diagonals of rational functions) were extremely simple pedagogical examples of differentially algebraic functions (see (13) and (18)). It is quite natural to imagine that, if the square-lattice Ising susceptibility is indeed a differentially algebraic function, it could correspond to quite a large polynomial differential equation (much more involved than Tutte's example (6) of Section 21), and that finding the polynomial relations associated with the reduction of the solution-series modulo primes and powers of primes would thus be quite difficult, their degree being very large. 
Along similar lines, it is worth recalling the hypergeometric example ${ }_{3} F_{2}\left([1 / 9,4 / 9,5 / 9],[1 / 3,1], 3^{6} x\right)$ introduced by G. Christol [20, a few decades ago, as an example of a holonomic $G$-series with integer coefficient $\$$ that may not be the diagonal of a rational function: after all these years, it is still an open question whether this function is, or is not, the diagonal of rational function! In such cases it is not guaranteed that the corresponding series modulo primes, or powers of primes, are algebraic functions. The analysis, modulo primes, and powers of primes, of such an example of a holonomic function is quite puzzling: in 24 it was shown (see Appendix B.4 in 24]), that it becomes extremely difficult to see whether a series like ${ }_{3} F_{2}\left([1 / 9,4 / 9,5 / 9],[1 / 3,1], 3^{6} x\right)$, is, modulo primes, an algebraic function or not, even for small prime numbers! This may be seen as a consequence of the fact that such a holonomic function is "extremely reluctant" to be seen as the diagonal of a rational function. Since we want to see differentially algebraic functions as a natural generalisation of diagonals of rational functions, we need to keep in mind that simple hypergeometric example: the difficulty we encounter in seeing the full susceptibility series reducing to algebraic functions modulo 3 (or modulo 5 , etc) could be a similar "reluctance".

\subsection{The 5043 terms series: no differentially algebraic equation?}

Let $F(x)$ be a generating function for which we have some finite number of known initial terms. Our algorithm searches for a polynomial

$$
P\left(x, y_{0}, y_{1}, y_{2}, \ldots, y_{k}\right)
$$

such that

$$
P\left(x, F(x), F^{\prime}(x), F^{\prime \prime}(x), \ldots, F^{(k)}(x)\right)=0 .
$$

Let $Q$ denote the left-hand side of the above equation. Our algorithm constructs a variety of potential forms $Q$ and for each checks if the known initial terms satisfy a functional equation of that form. Each possible $Q$ is a sum of terms of the form

$$
p_{i}(x) \cdot(F(x))^{c_{i}(0)} \cdot\left(F^{\prime}(x)\right)^{c_{i}(1)} \cdots\left(F^{(k)}(x)\right)^{c_{i}(k)},
$$

where $p_{i}(x)$ is called the polynomial coefficient and the rest is called the functional term. Clearly, each possible functional term can be thought of as a weak composition (that is, its summands may be zero), $c_{i}=\left(c_{i}(0), c_{i}(1), \ldots, c_{i}(k)\right)$, and so each form $Q$ is associated with some set of compositions, along with the degrees of the polynomial coefficients.

Each form $Q$ to which we attempt to fit our known initial terms is uniquely specified by a 3 -tuple $(m, k, d)$. Here, $d$ is the maximum degree of all polynomial coefficients. The set of functional terms corresponds to the set of all compositions of $m$ into exactly $k+1$ parts. For example, $(m, k, d)=(3,1, d)$ corresponds to the set of compositions

$$
\{(0,0),(1,0),(0,1),(2,0),(1,1),(0,2),(3,0),(2,1),(1,2),(0,3)\},
$$

and thus the functional terms of this particular $Q$ are

$$
\begin{array}{lllll}
1 & F(x) & F^{\prime}(x) & F(x)^{2} & F(x) F^{\prime}(x) \\
F^{\prime}(x)^{2} & F(x)^{3} & F(x)^{2} F^{\prime}(x) & F(x) F^{\prime}(x)^{2} & F^{\prime}(x)^{3} .
\end{array}
$$

$\dagger$ The fact that the corresponding series expansion is a series with integer coefficients is far from obvious (see appendix D in [20]). 
The quantity $m$ is called the algebraic order, while $k$ is the differential order. Using the known initial terms for $F(x)$, we calculate the functional terms of each component of the predicted form. The polynomial coefficients represent the unknowns to be determined. Each has the form

$$
p_{i}(x)=a_{i, d} \cdot x^{d}+a_{i, d-1} \cdot x^{d-1}+\cdots+a_{i, 1} \cdot x+a_{i, 0},
$$

and therefore if the predicted form has $R$ components, the total number of unknown quantities is $R \cdot(d+1)$. As we are seeking a polynomial $P$ such that $P\left(x, F(x), \cdots, F^{(k)}(x)\right)=0$, the predicted form can be expanded to give a series in $x$ in which each coefficient must equal zero. So long as a sufficient number of initial terms of $F(x)$ are provided, we can attempt to solve the system. When the system involves $R \cdot(d+1)$ unknowns, we provide $R \cdot(d+1)+T$ equations in these variableswe typically use $T=10$. This provides some confidence that a conjectured algebraic differential equation is in fact satisfied by the unknown series.

The procedure to check whether the known initial terms of a series satisfy an algebraic differential equation now proceeds as follows. Given $N$ known initial terms, we first calculate all tuples $(m, k, d)$ for which the number of unknowns $U$ is at most $N-k-T$. Each time a derivative of the known initial terms is computed, the number of unknowns needed increases by one. This is why we require $U+k \leq N-T$. Some of these predicted forms are properly contained in others, e.g. there is no need to check for a predicted form $(m, k, d)=(3,1,5)$ if we intend to also check $(m, k, d)=(3,2,7)$. Therefore, we filter out all such redundancies. What remains is a list of maximal predicted forms for which there are a sufficient number of known initial terms to check. For each, we construct the components, extract the linear system, and attempt to find a solution.

To demonstrate the method, consider the sequence defined by

$$
h_{n+2}=\sum_{i=1}^{n} i \cdot h_{i+1} h_{n-i+2},
$$

with initial conditions $h_{0}=h_{1}=0$ and $h_{2}=1$ (see Appendix D.2 for more about this sequence). The first few term of the power series of this sequence are

$$
F(x)=x^{2}+x^{3}+3 x^{4}+14 x^{5}+85 x^{6}+\cdots,
$$

and it is computationally easy to get thousands of known initial terms. If we provide at least 35 initial terms to our algorithm (using $T=10$ ), it eventually reaches the form $Q$ corresponding to $(m, k, d)=(2,1,3)$. This corresponds to checking if $F(x)$ satisfies a functional equation of the form

$$
\begin{aligned}
p_{0}(x)+ & p_{1}(x) \cdot F(x)+p_{2}(x) \cdot F^{\prime}(x)+p_{3}(x) \cdot F(x)^{2} \\
& +p_{4}(x) \cdot\left(F^{\prime}(x)\right)^{2}+p_{5}(x) \cdot F(x) F^{\prime}(x)=0,
\end{aligned}
$$

where each $p_{i}(x)$ has degree at most 3 . Into this equation we substitute the first 35 terms of the power series of $F(x)$. Upon evaluating $F^{\prime}(x)$ and computing the product $F(x) F^{\prime}(x)$, we are left with a power series on the left-hand side involving the unknown coefficients of each of the polynomials $p_{i}(x)$. As each term of this power series must equal zero, we can attempt to find a solution for the unknown coefficients of the $p_{i}(x)$ by solving a linear system where each equation is a coefficient of the left-hand side. This system has 34 equations and 24 unknowns, and has a solution corresponding to

$$
p_{0}(x)=x^{3}, \quad p_{1}(x)=-x, \quad p_{2}(x)=0, \quad p_{3}(x)=-1, \quad p_{4}(x)=0, \quad p_{5}(x)=x .
$$


Therefore, we have used 35 initial terms of $F(x)$ to conjecture that $F(x)$ satisfies the equation

$$
x^{3}-x \cdot F(x)-F(x)^{2}+x \cdot F(x) F^{\prime}(x)=0 .
$$

Our implementation performs all of these calculations in under a second.

\subsection{Running the program: some simple examples.}

5.3.1. Running the program: a first simple pullback hypergeometric example.

We first consider a simpler example of composition than that of two ${ }_{2} F_{1}$ hypergeometric functions given in Section (4.2): rather we consider the composition of a ${ }_{2} F_{1}$ hypergeometric function with $x \ln (1+x)$ :

$$
F(x)={ }_{2} F_{1}\left(\left[\frac{1}{2}, \frac{1}{2}\right],[1], 16 x \ln (1+x)\right) .
$$

This function has an irregular singularity at $x=-1$. Using our program with more than 2407 coefficients one can find (in 4834 seconds) one non-linear differential equation (29) for this differentially algebraic function, of order three, of degree 18 in $x$, and degree 5 in $F(x), F^{\prime}(x), F^{\prime \prime}(x)$ and $F^{\prime \prime \prime}(x)$. Note that

$$
\begin{aligned}
F(x) & ={ }_{2} F_{1}\left(\left[\frac{1}{2}, \frac{1}{2}\right],[1], 16 x \ln (1+x)+16 \alpha x\right) \\
& =1+4 \alpha \cdot x+\left(36 \alpha^{2}+4\right) \cdot x^{2}+\left(400 \alpha^{3}+72 \alpha-2\right) \cdot x^{3}+\cdots
\end{aligned}
$$

is also a solution of the same non-linear differential equation (29). This one-parameter family of solutions (35) is a consequence of the fact that, for compositions of holonomic functions, there always exists a non-linear differential equation (29) that inherits the (differential Galois group) symmetries of the underlying holonomic functions see for instance [46]. This one-parameter family of solutions (35) clearly illustrates that the solutions of the associated non-linear differential equation (29) have movable singularities corresponding to $16 x \ln (1+x)+16 \alpha x=1$ which depend on the parameter $\alpha$ of the initial conditions of the series (35).

5.3.2. Running the program: a second simpler example of composition of holonomic functions.

We further illustrate these questions of symmetries, differential Galois groups, families of solutions and movable singularities with another simple example of the composition of holonomic functions $F(x)=f(g(x))$, where

$$
f(x)=\frac{1}{1-x}, \quad g(x)=x \ln (1+x), \quad f(g(x))=\frac{1}{1-x \ln (1+x)} .
$$

The two functions $f(x)$ and $g(x)$ are solutions of order-one and order-two linear differential operators, $(x-1) D_{x}+1$ and $(x+2)-x(x+2) D_{x}+x^{2}(1+x) D_{x}^{2}$, which have, respectively, the one and two-parameter families of solutions:

$$
f(\alpha ; x)=\frac{\alpha}{1-x}, \quad g(\beta, \gamma ; x)=\beta \cdot x \cdot \ln (1+x)+\gamma \cdot x .
$$

Our program very quickly gives a non-linear differential equation $\mathcal{N}_{1}=0$, for $F(x)=f(g(x))$ given by (36), where $\mathcal{N}_{1}$ reads:

$$
\mathcal{N}_{1}=(1+x) \cdot F(x)-\left(x^{2}+x+1\right) \cdot F(x)^{2}+x \cdot(1+x) \cdot F^{\prime}(x),
$$


and one readily verifies that

$$
\begin{aligned}
& f(1 ; g(1, \gamma ; x))=\frac{1}{1-x \cdot \ln (1+x)-\gamma x} \\
& \quad=1+\gamma \cdot x+\left(\gamma^{2}+1\right) \cdot x^{2}+\left(2 \gamma+\gamma^{3}-\frac{1}{2}\right) \cdot x^{3}+\cdots
\end{aligned}
$$

is a solution of (38).

Note that

$$
F(x)=f(\alpha ; g(\beta, \gamma ; x))=\frac{\alpha}{1-\beta x \cdot \ln (1+x)-\gamma x} .
$$

is not a solution of (38) in general: it is a solution only when $\beta=\alpha=1$.

Actually, if one looks for the power series solutions of the non-linear differential equation (38), $F(x)=a_{0}+a_{1} x+a_{2} x^{2}+\cdots$, one finds immediately the condition $a_{0}\left(a_{0}-1\right)=0$. If $a_{0}=1$ one gets, order by order, the one-parameter series (39), when $a_{0}=0$ gives the null function $F(x)=0$.

There is another non-linear differential equation $\mathcal{N}_{2}=0$, where $\mathcal{N}_{2}$ reads

$$
\begin{aligned}
& \mathcal{N}_{2}=(x+2) \cdot F(x)^{2}+x \cdot(x+2) \cdot F(x) \cdot F^{\prime}(x) \\
& \quad+2 \cdot(1+x) \cdot\left(x^{2}+x+1\right) \cdot F^{\prime}(x)^{2}-(1+x) \cdot\left(x^{2}+x+1\right) \cdot F(x) \cdot F^{\prime \prime}(x),
\end{aligned}
$$

which is homogeneous (quadratic) in $F(x), F^{\prime}(x)$ and $F^{\prime \prime}(x)$. This homogeneity is inherited from the (differential Galois group) symmetry of the order-one operator $(x-1) D_{x}+1$. One readily verifies that $f(\alpha ; g(1, \gamma ; x))$ is also a solution of (41). Again if one looks for the power series solutions of the non-linear differential equation (41), one finds, order by order, that either $F(x)=0$ or $F(x)=f(\alpha ; g(1, \gamma ; x))$.

There is a third non-linear differential equation $\mathcal{N}_{3}=0$, where $\mathcal{N}_{3}$ reads:

$$
\begin{aligned}
\mathcal{N}_{3}= & (x+2) \cdot(F(x)-1) \cdot F(x)^{2}-x \cdot(x+2) \cdot F(x) \cdot F^{\prime}(x) \\
& -2 x^{2} \cdot(1+x) \cdot F^{\prime}(x)^{2}+x^{2} \cdot(1+x) \cdot F(x) \cdot F^{\prime \prime}(x),
\end{aligned}
$$

which has as a solution $f(1 ; g(\beta, \gamma ; x))$. This time the (non-homogeneous) non-linear differential equation (42) inherits the (differential Galois group) symmetry of the ordertwo operator $(x+2)-x(x+2) D_{x}+x^{2}(1+x) D_{x}^{2}$. If one looks for the power series solutions of the non-linear differential equation (42), one immediately obtains the condition $a_{0}^{2}\left(a_{0}-1\right)=0$. The condition $a_{0}=0$ yields, order by order, $F(x)=0$. The condition $a_{0}=1$ yields, order by order, the two-parameter family of solutions $F(x)=f(1 ; g(\beta, \gamma ; x))$.

Finally there is a last non-linear differential equation $\mathcal{N}_{4}=0$, where $\mathcal{N}_{4}$ reads:

$$
\begin{aligned}
& \mathcal{N}_{4}=6 \cdot x^{2} \cdot(x+2)(1+x) \cdot F(x)^{2} F^{\prime}(x)^{3}-2 x^{2} \cdot(x+3) \cdot F(x)^{3} F^{\prime}(x)^{2} \\
& \quad-6 \cdot x^{2} \cdot(x+2) \cdot(1+x) \cdot F(x)^{3} F^{\prime}(x) F^{\prime \prime}(x)+x^{2} \cdot(x+3) \cdot F(x)^{4} F^{\prime \prime}(x) \\
& \quad+x^{2} \cdot(x+2) \cdot(1+x) \cdot F(x)^{4} F^{\prime \prime \prime}(x),
\end{aligned}
$$

which is homogeneous (quintic) in $F(x), F^{\prime}(x), F^{\prime \prime}(x)$ and $F^{\prime \prime \prime}(x)$ and only this nonlinear differential equation has the three-parameter family (40) of solutions inherited from the one and two-parameter families of solutions (37) of the order-one and ordertwo linear differential operators (i.e. inherited from the differential Galois groups of the order-one and order-two linear differential operators). If one looks for the power series solutions of the non-linear differential equation (43), one immediately obtains the two conditions $a_{0}=0$ and $2 a_{0}^{2} a_{3}+a_{2} a_{0}\left(a_{0}-4 a_{1}\right)+a_{1}^{2}\left(2 a_{1}-a_{0}\right)=0$. Condition $a_{0}=0$ gives the null function $F(x)=0$, when the second condition gives, order by order, the three-parameter series (40). 
There are some relations between the non-linear ODEs, for instance:

$$
\begin{aligned}
\mathcal{N}_{3}= & x \cdot F(x) \cdot \frac{d \mathcal{N}_{1}}{d x}-2 \cdot\left(F(x)+x \cdot F^{\prime}(x)\right) \cdot \mathcal{N}_{1}, \\
x \cdot \mathcal{N}_{2}= & -\left(x^{2}+x+1\right) \cdot F(x) \cdot \frac{d \mathcal{N}_{1}}{d x} \\
& \quad+\left((2 x+1) \cdot F(x)+\left(x^{2}+x+1\right) \cdot F^{\prime}(x)\right) \cdot \mathcal{N}_{1} .
\end{aligned}
$$

or

$$
\mathcal{N}_{4}=(x+2) \cdot F(x)^{3} \cdot \frac{d \mathcal{N}_{3}}{d x}-F(x)^{2} \cdot\left(F(x)+3 \cdot(x+2) \cdot F^{\prime}(x)\right) \cdot \mathcal{N}_{3}
$$

If $\mathcal{N}_{1}=0$ necessarily $\mathcal{N}_{2}=0$ and $\mathcal{N}_{3}=0$. Similarly, if $\mathcal{N}_{3}=0$ necessarily $\mathcal{N}_{4}=0$ (but not conversely).

All these non-linear differential equations (38), (41), (42), (43) share the same solution (36), and have movable singularities.

In contrast with linear differential equations one can have, for a given series, many non-linear differential equations of the same order, for instance $\mathcal{N}_{2}=0$ and $\mathcal{N}_{3}=0$. For linear differential equations, the (unique) minimal order linear ODE requires (paradoxically [12]) many more coefficients to be obtained from the so-called "guessing procedures" than higher order ODEs. When we use our (non-linear guessing) program it is not clear if the minimal order non-linear ODEs are the easiest to be obtained, requiring the minimal number of coefficients to be "guessed". Furthermore, higher order ODEs could be of smaller "size".

5.3.3. Running the program: a third simple example of a ratio of holonomic functions.

The non-linear differential equation (29) corresponding to the simple example given in 24] of the ratio of two hypergeometric functions

$$
R(x)=\frac{{ }_{2} F_{1}\left(\left[\frac{1}{3}, \frac{1}{3}\right],[1], 27 x\right)}{{ }_{2} F_{1}\left(\left[\frac{1}{2}, \frac{1}{2}\right],[1], 16 x\right)},
$$

is obtained with our program in about 5 seconds. Note that this non-linear differential equation (see equation (D.2) in Appendix D of [24]) can be rewritten in a form that makes the emergence of a Schwarzian derivative crystal clear:

$$
\begin{aligned}
& 2 x^{2} \cdot(1-27 x)^{2} \cdot(1-16 x)^{2} \cdot R_{1}{ }^{2} \cdot\left(\frac{R_{3}}{R_{1}}-\frac{3}{2} \frac{R_{2}{ }^{2}}{R_{1}{ }^{2}}\right) \\
& \quad+2 x^{2} \cdot(1-16 x) \cdot(1-27 x) \cdot(1+72 x) \cdot R \cdot R_{2} \cdot\left(3 \frac{R_{1}}{R}-\frac{R_{3}}{R_{2}}\right) \\
& \quad+(1-16 x) \cdot\left(1944 x^{3}-1569 x^{2}+58 x-1\right) \cdot R_{1}{ }^{2} \\
& \quad-2 \cdot x \cdot\left(93312 x^{3}-168 x^{2}-297 x+4\right) \cdot R \cdot R_{2} \\
& \left.\quad-2 \cdot\left(29376 x^{3}+5580 x^{2}-221 x+1\right) \cdot R \cdot R_{1}+44 x^{2}-432 x+1\right) \cdot R^{2}=0 .
\end{aligned}
$$

5.3.4. Running the program: a fourth simple example of a ratio of holonomic functions.

The non-linear differential equation of the form (29) for a ratio of ${ }_{4} F_{3}$ and ${ }_{2} F_{1}$ hypergeometric functions such as

$$
R(x)=\frac{{ }_{4} F_{3}\left(\left[\frac{1}{2}, \frac{1}{2}, \frac{1}{2}, \frac{1}{2}\right],[1,1,1], 256 x\right)}{{ }_{2} F_{1}\left(\left[\frac{1}{2}, \frac{1}{2}\right],[1], 16 x\right)},
$$


is obtained in 2834 seconds by our program. The non-linear differential equation (29) is of order five, and of degree 12 in $x$ and is a (quadratic) homogeneous polynomial in $R(x)$, and its derivatives $R^{\prime}(x), \ldots, R^{(5)}$.

\subsubsection{Running the program: the composition of holonomic functions.}

The composition of two ${ }_{2} F_{1}$ hypergeometric functions, given in Section (4.2), is the solution of a non-linear differential equation (29) of order four, and of degree 32 in $x$ and of degree at most $m=11$ in $F(x)$, and its derivatives $F^{\prime}(x), \ldots, F^{(4)}$. With $m=11$, there are $\left(\begin{array}{c}m+4 \\ 4\end{array}\right)=1365$ monomials $F(x)^{c_{i}(0)} F^{\prime}(x)^{c_{i}(1)} \cdots F^{(4)}(x)^{c_{i}(4)}$ of degree exactly $d_{c}=c_{i}(0)+c_{i}(1)+c_{i}(2)+c_{i}(3)+c_{i}(4)=11$, in $F(x)$, and its derivatives $F^{\prime}(x), \ldots, F^{(4)}$. There are $\left(\begin{array}{c}10+4 \\ 4\end{array}\right)=1001$ monomials of degree $d_{c}$ exactly equal to $10,\left(\begin{array}{c}9+4 \\ 4\end{array}\right)=715$ monomials of degree $d_{c}$ exactly equal 9 , etc., and therefore there are $\left(\begin{array}{c}11+4 \\ 4\end{array}\right)+\left(\begin{array}{c}10+4 \\ 4\end{array}\right)+\left(\begin{array}{c}9+4 \\ 4\end{array}\right)+\cdots=\left(\begin{array}{c}11+5 \\ 5\end{array}\right)=4368$ monomials of degree less than or equal to $m=11$.

Since the degree in $x$ is 32 the composition of two ${ }_{2} F_{1}$ hypergeometric functions given in Section (4.2) would require a series expansion with $4368 \cdot 33=144144$ coefficients to find the non-linear differential equation (29). Another program specially built to detect non-linear differential equations (29) that are homogeneous polynomials in $F(x)$, and its derivatives $F^{\prime}(x), \ldots, F^{(4)}$ would require $1365 \cdot 33=45045$ coefficients to find the non-linear differential equation.

In general finding the non-linear differential equation (29) of order $k$, of degree $d$ in $x$, of degree at most $m$ in $F(x)$, and its derivatives $F^{\prime}(x), \ldots, F^{(k)}$ would require

$$
(d+1) \cdot\left(\begin{array}{c}
m+k+1 \\
k+1
\end{array}\right)
$$

coefficients to be found with our program.

Finding the non-linear differential equation (16) solving the example of an algebraic function of holonomic functions (14), displayed in Section (4.1), would require a really large number of series coefficients.

\section{Application to the Ising series.}

We applied the algorithm described above to the 5043 known initial terms of the square-lattice Ising susceptibility series, using both the high- and low-temperature series. As the generating functions for the two Ising series are believed to have a natural boundary, they cannot be algebraic or D-finite. This allows us to eliminate any forms $(m, k, d)$ with $k=0$ or $m=1$. This restriction leaves 133 possible polynomials $P\left(x, y_{0}, \ldots, y_{k}\right)$.

Using approximately 25 hours of computation time on a machine with 256 gigabytes of RAM, we have determined that neither the high- nor low-temperature square-lattice Ising susceptibility series satisfy an algebraic differential equation corresponding to any possible predicted form, as described above, with $U+k+T \leq$ 5043. We repeated the computation with the series obtained by shifting the coefficients of the two given series one, two, and three positions (i.e., dividing by $x, x^{2}$, and $x^{3}$ ), because the number of terms needed to guess a functional equation is very sensitive to the positioning of the first non-zero term of the series.

This provides a collection of negative results. For example, if the susceptibility series are differentially algebraic with algebraic and differential order 3 (i.e. $m=3$ 
and $k=3$ ), then it must be that the polynomial coefficients have degree strictly greater than 142 , otherwise our algorithm would have found the algebraic differential equations that they satisfy.

\section{Conclusion: To be or not to be differentially algebraic.}

The preceding examples allow us to better understand the class of power series with integer coefficients, no Hadamard gaps, and non-zero radius of convergence, that reduce to algebraic functions modulo $p^{r}$. This is an extremely large class of functions probably with a closure property with respect to the composition of functions. The question whether this class of functions reduces to differentially algebraic functions remains a (difficult) open question. It contains the class of functions generated by the composition of algebraic functions of diagonals of rational functions, these functions always being differentially algebraic.

In summary, the question that is raised is if the full susceptibility of the Ising model (and more generally other non-holonomic functions emerging in lattice statistical mechanics, or enumerative combinatorics [24) is differentially algebraic or is differentially transcendental: If the full susceptibility of the Ising model is differentially transcendental, the reduction modulo $2^{r}$ of the full susceptibility series to algebraic functions seen in 15, should just be seen as a consequence of a specific character of powers of two for the $\chi^{(n)}$, each of these $n$-fold integrals being series with integer coefficients up to an overall $2^{r}$ factor. This is already a remarkable property (see 24]). A differentially transcendental full susceptibility has, of course, no reason to reduce to algebraic functions modulo other primes, or powers of primes. However, one would now have to understand how such a differentially transcendental full susceptibility series could actually correspond to "algorithmic integrability", these series being obtained from an iterative algorithm having polynomial growth!

That is to say, the susceptibility series coefficients $c_{n}$ are obtained from an algorithm with polynomial growth which is a consequence of a quadratic recurrence relation [47, 48, 49] on the two-point correlation functions $C(M, N)$ (recursion on the two integers $M$ and $N$ ). It is also tempting to speculate on the existence of a nonlinear recursion, or more generally a polynomial relation on a fixed finite number $p+1$ of coefficients, of the type:

$$
P\left(n, c_{n}, c_{n-1}, c_{n-2}, \cdots c_{n-p}\right)=0 .
$$

Such a non-linear recursion should be compared with the non-linear ODE (A.1) defining a differentially algebraic function. One can always convert a linear recurrence (with a finite number of terms) into a linear differential equation: in the linear case, there is a "duality" between these two concepts. In contrast the correspondence between non-linear recursions and non-linear ODEs is more involved. Non-linear ODEs yield non-linear recursions, but these recursions are, not, in general, relations on a fixed number of terms like (51). For instance, Tutte's non-linear ODE (6) yields a quadratic recursion (D.1) where the number of terms involved grows at each step. Conversely, the existence of a non-linear recursion of the form (51) is not a guarantee that the corresponding power series is differentially algebraic. One can imagine the existence of a non-linear recursion of the form (51) for the susceptibility series that could be obtained quite reasonably, while at the same time the non-linear

$\ddagger$ A function that is not differentially algebraic is called differentially transcendental. 
ODE (2) proving the differentially algebraic character of the susceptibility series being extremely difficult to identify (if it exists!).

It now becomes useful to build more efficient programs (see Section [5.2) to see when such very long series, with more than 5043 coefficients, are differentially algebraic, or correspond to a non-linear recursion such as (51). Recalling the previous examples (14), (19), for which the polynomial differential equations were, even for these extremely simple examples, quite large polynomials, sometimes of quite high degrees, one can imagine, if the full susceptibility of the Ising model turns out to be differentially algebraic, that the corresponding polynomial differential equation might be quite a large expression (recall the 144144 coefficients required to find the non-linear differential equation (29) for a simple composition of two ${ }_{2} F_{1}$ hypergeometric function). In the most pessimistic scenario such programs (that could be programs modulo primes) should, at least, give some lower bounds for the various degrees of the polynomial. Actually we already have a first modulo prime version of our program. We checked with 5043 terms modulo 3 and, similarly, did not find any differentially algebraic equation modulo 3. Determining if the square-lattice Ising susceptibility is a differentially algebraic function is clearly a serious challenge. In contrast, non-holonomic generating functions of examples from enumerative combinatorics [50, 51, 52, 53, 54, 55] may be a much simpler testing ground for such programs.

Acknowledgments: JMM would like to thank J-P. Allouche, C. Banderier, G. Christol, S. Fischler, T. Rivoal, J. Rocques and J. Sauloy J-A. Weil for fruitful discussions on differentially algebraic functions. JMM would like to thank D. Bertrand for providing a proof by Ehud Hrushovski, on the closure by composition of differentially algebraic functions. He also thanks C. Koutschan and M. Mezzarobba for discussions on zeros of D-finite functions. This work has been performed without any support of the ANR, the ERC, the MAE or any PES of the CNRS. AJG and IJ would like to thank the Australian Research Council for its support through grant DP140101110. IJ was supported by an award under the Merit Allocation Scheme of the NCI National facility at the ANU, where the bulk of the large scale numerical computations were performed. JP would like to thank the Centre of Excellence for Mathematics and Statistics of Complex Systems for their hospitality in June 2015, where this research was partially conducted. JP was supported by the National Science Foundation and the Australian Academy of Science under award \#1514825.

\section{Appendix A. Differentially Finite and Differentially Algebraic Series}

Let $\mathbb{K}$ be a field of characteristic zero (usually $\mathbb{K}=\mathbb{Q}$ or $\mathbb{K}=\mathbb{C}$ ).

Appendix A.1. D-finite or holonomic functions

A function or formal power series $f(x) \in \mathbb{K}[[x]]$ satisfying a linear differential equation

$$
a_{n}(x) \cdot f^{(n)}(x)+a_{n-1}(x) \cdot f^{(n-1)}(x)+\ldots+a_{0}(x) \cdot f(x)=0,
$$

with polynomials $a_{i}(x) \in \mathbb{K}[[x]]$ is called differentiably finite or D-finite or holonomic. The set of all D-finite power series is closed under addition, multiplication, right composition with algebraic functions, Laplace Borel transform or inverse Borel transform. Lipshitz [21] proved that the diagonal of a D-finite power series in several 
variables is D-finite, from which follows that the Hadamard product of a rational power series with a $\mathrm{D}$-finite power series is $\mathrm{D}$-finite.

A function that can be expressed as the diagonal of a rational function is D-finite. The converse is false: for instance, a D-finite function with an irregular singularity, such as $\exp (-1 /(1-x))$, is a counter example.

A function that is algebraic modulo a prime $p$ or a power of a prime $p^{r}$ may be D-finite, but need not be. It also may be expressible as the diagonal of a rational function.

The ratio of two (non-algebraic) holonomic functions is generically not holonomic: it is differentially algebraic. The composition of two non-algebraic holonomic functions is not holonomic, though it is differentially algebraic.

\section{Appendix A.2. Differentially algebraic functions}

Let $P$ be a non-zero polynomial in $n+2$ variables with coefficients in a field of characteristic zero $\mathbb{K}$, such that

$$
P\left(x, f(x), f^{\prime}(x), \ldots, f^{(n)}(x)\right)=0 .
$$

This is called an algebraic differential equation, and the function $f(x) \in \mathbb{K}[[x]]$ satisfying the algebraic differential equation is called differentially algebraic or Dalgebraic. Lipshitz and Rubel 32 proved that the set of all D-algebraic power series is closed under addition, subtraction, multiplication and division (suitably restricted so that the expressions are sensible) and composition of functions. This set is not, however, closed under Hadamard products, and it is not closed under the Laplace Borel transform or the inverse Borel transform. One simple example is, for instance, the Borel transform of the generating function of the Bell numbers.

Formal series solutions of differentially algebraic equations are necessarily Gevrey series [56] (see also the first footnote in Yves André survey [57]) i.e. the growth of the coefficients is bounded by $C^{n} \cdot(n !)^{s}$. Conversely, if a series is "too divergent" (not Gevrey) it cannot be differentially algebraic, it is differentially transcendent.

Appendix A.3. Diagonals of rational functions

A power series in $k$ variables is written

$$
F\left(x_{1}, x_{2}, \cdots, x_{k}\right)=\sum_{m_{1}} \sum_{m_{2}} \cdots \sum_{m_{k}} a_{m_{1}, m_{2}, \cdots, m_{k}} \cdot x_{1}^{m_{1}} x_{1}^{m_{2}} \cdots x_{k}^{m_{k}} .
$$

Its diagonal is the one-variable series

$$
\operatorname{Diag}\left(F\left(x_{1}, x_{2}, \cdots, x_{k}\right)\right)=\sum_{m} a_{m, m, \cdots, m} \cdot x^{m} .
$$

Now if $F\left(x_{1}, x_{2}, \cdots, x_{k}\right)=P\left(x_{1}, x_{2}, \cdots, x_{k}\right) / Q\left(x_{1}, x_{2}, \cdots, x_{k}\right)$ where $P$ and $Q$ are polynomials with rational coefficients, and with $Q(0,0, \cdots, 0) \neq 0$, then $F\left(x_{1}, x_{2}, \cdots, x_{k}\right)$ is a rational function, and $\operatorname{Diag}\left(F\left(x_{1}, x_{2}, \cdots, x_{k}\right)\right)$ is referred to as the diagonal of a rational function.

\section{Appendix A.4. Global boundedness}

A power series $\sum a_{n} x^{n}$ with rational coefficients $a_{n}$ and non-zero radius of convergence is said to be globally bounded if there exists an integer $N$ such that the series can be recast as one with integer coefficients by a rescaling $x \rightarrow N x$. 
Diagonals of rational functions, or diagonals of algebraic functions, are globally bounded series. Algebraic functions, being diagonals of rational functions [58, are globally bounded series.

Appendix A.5. Some relevant theorems

- Eisenstein's Theorem [59] Chapter 3, 139. If a power series with rational coefficients represents an algebraic function it is globally bounded: it can be recast into a series which has integer coefficients.

- 59 Chapter 3,163 . If a power series with integer coefficients represents a rational function, then, after a certain number of terms, the coefficients modulo $m(m$ arbitrary), form a periodic sequence (this result cannot be extended to algebraic functions).

- 59. Chapter 3,164 . The algebraic function $1 / \sqrt{1-4 x}$ on expansion gives a power series with integer coefficients. These coefficients are for no odd prime number $p$ periodic modulo $p$.

- 59 Chapter 3,165 . The radius of convergence of a non-terminating power series with integer coefficients is at most 1.

\section{Appendix B. Solutions of Tutte's differentially algebraic equation (6) modulo primes, for miscellaneous values of $q$.}

Let us consider the series (3) divided by $q(q-1)$ :

$$
\frac{H(x)}{q(q-1)}=x^{2}+(q-2) \cdot \sum_{n=3}^{\infty} P_{n}(q) \cdot x^{n} .
$$

- The reduction modulo 3 of this (normalised) series (B.1) for $q=7$ is an algebraic function:

$$
F(x)^{3}+2 x^{2} \cdot F(x)+x^{4} \cdot\left(1+2 x+x^{2}+x^{5}\right)=0 .
$$

The reduction modulo 7 of the $q=7$ series $(\mathbb{B} .1)$ is the solution (modulo 7 ) of a polynomial equation of degree 15 in $F(x)$, and degree 27 in $x$. Modulo 11 the $q=7$ series (B.1) is the solution of a polynomial equation of degree 11 in $F(x)$, and degree 19 in $x$. Modulo 13 the $q=7$ series (B.1) is a solution of a polynomial equation of degree 15 in $F(x)$, and degree 27 in $x$.

- The reduction modulo 5 of the $q=11$ series (B.1) satisfies (modulo 5 )

$$
\begin{aligned}
\left(x^{25}\right. & +4) \cdot F(x)^{4}+x \cdot\left(2 x^{25}+3\right) \cdot F(x)^{3} \\
& +x^{2} \cdot\left(4 x^{25}+1\right) \cdot F(x)^{2}+x^{3} \cdot\left(3 x^{25}+2\right) \cdot F(x) \\
& +3 x^{5}+x^{6}+4 x^{7}+3 x^{8}+2 x^{9}+x^{10}+3 x^{11}+3 x^{12}+4 x^{13}+x^{14}+4 x^{15} \\
& +2 x^{17}+3 x^{18}+4 x^{19}+2 x^{20}+4 x^{21}+3 x^{23}+2 x^{24}+2 x^{25}+4 x^{26}+3 x^{27} \\
& +3 x^{28}+2 x^{30}+4 x^{32}+3 x^{33}+x^{34}+x^{35}+3 x^{36}+4 x^{37}+x^{38}+x^{40}=0 .
\end{aligned}
$$

The $q=11$ series (B.1) satisfies modulo 7

$$
\begin{aligned}
F(x)^{4} & +(5 x+1) \cdot F(x)^{3}+x \cdot\left(6 x^{2}+5 x+2\right) \cdot F(x)^{2} \\
& +x^{2} \cdot\left(x^{2}+2 x+6\right) \cdot F(x)+x^{4} \cdot\left(1+5 x+2 x^{2}\right)=0 .
\end{aligned}
$$

- For the $q=-1$ series, modulo 5 the series (B.1) is an algebraic function

$$
2 x^{3} \cdot\left(2+x+x^{2}\right)+x \cdot F(x)+F(x)^{2}=0 .
$$


Modulo 7 the $q=-1$ series is also an algebraic function

$$
\begin{aligned}
F(x)^{5} & +\left(6 x^{2}+x+3\right) \cdot F(x)^{4}+\left(2 x^{4}+5 x^{2}+6 x+1\right) \cdot F(x)^{3} \\
& +x \cdot\left(6 x^{4}+5 x^{3}+4 x^{2}+x+3\right) \cdot F(x)^{2} \\
& +x^{2} \cdot\left(x^{5}+6 x^{4}+5 x^{3}+5 x^{2}+2\right) \cdot F(x) \\
& +x^{4} \cdot\left(5+3 x+3 x^{2}+x^{3}+2 x^{4}+4 x^{5}\right)=0 .
\end{aligned}
$$

Modulo 11 the $q=-1$ series is the solution of a polynomial equation of degree 7 in $F(x)$, and degree 13 in $x$. Modulo 13 the $q=-1$ series is the solution of a polynomial equation of degree 6 in $F(x)$, and degree 10 in $x$. Modulo 17 the $q=-1$ series is the solution of a polynomial equation of degree 9 in $F(x)$, and degree 15 in $x$. function

- For the $q=1 / 2$ series (B.1) (when it work $(\mathbb{E}$ ), we find, modulo 5 , the algebraic

$$
F(x)^{2}+(x+1) \cdot(2 x+1) \cdot F(x)+x^{2} \cdot\left(4+x+x^{2}\right)=0,
$$

and modulo 7

$$
\begin{aligned}
& F(x)^{4}+(5 x+1) \cdot F(x)^{3}+x \cdot\left(6 x^{2}+5 x+2\right) \cdot F(x)^{2} \\
& \quad+x^{2} \cdot\left(x^{2}+2 x+6\right) \cdot F(x)+x^{4} \cdot\left(1+5 x+2 x^{2}\right)=0 .
\end{aligned}
$$

Modulo 11 the $q=1 / 2$ series is the solution of a polynomial equation of degree 7 in $F(x)$, and degree 13 in $x$. Modulo 13 the $q=1 / 2$ series is the solution of a polynomial equation of degree 15 in $F(x)$, and degree 27 in $x$. Modulo 17 the $q=1 / 2$ series is the solution of a polynomial equation of degree 9 in $F(x)$ and degree 15 in $x$. Modulo 23 the $q=1 / 2$ series is the solution of a polynomial equation of degree 15 in $F(x)$, and degree 25 in $x$.

\section{Appendix C. A simple generalisation of Tutte's differentially algebraic equation (6).}

The two-parameter differentially algebraic equation (the two parameters are the two integers $M$ and $N$ ):

$$
\begin{aligned}
& -2 M^{2} \cdot(M-N) \cdot x+\left(M \cdot x+10 H(x)-6 x \frac{d H(x)}{d x}\right) \cdot \frac{d^{2} H(x)}{d x^{2}} \\
& -M \cdot(M-4 N) \cdot\left(20 H(x)-18 x \frac{d H(x)}{d x}+9 x^{2} \frac{d^{2} H(x)}{d x^{2}}\right)=0,
\end{aligned}
$$

has solution series of the form

$$
M \cdot(M-N) \cdot x^{2}+\sum_{n=3}^{\infty} M \cdot(M-N) \cdot(M-2 N) \cdot P_{n}(M, N) \cdot x^{n}+\cdots
$$

where:

$$
\begin{aligned}
& P_{3}(M, N)=1, \quad P_{4}(M, N)=4 M-9 N, \quad P_{5}(M, N)=8 M^{2}-37 M N+43 N^{2}, \\
& P_{6}(M, N)=176 M^{3}-1245 M^{2} N+2951 M N^{2}-2344 N^{3}, \\
& P_{7}(M, N)=1456 M^{4}-13935 M^{3} N+50273 M^{2} N^{2}-81036 M N^{3}+49248 N^{4},
\end{aligned}
$$

where the $P_{n}$ 's are homogeneous polynomials of $M$ and $N$ of degree $n-3$. One can show that all these two-parameter series with integer coefficients reduce to algebraic functions modulo every prime, or power of a prime.

$\S$ These calculations cannot be performed modulo any integer. 


\section{Appendix D. Miscellaneous differentially algebraic equations associated with simple quadratic recursions.}

Recall that $H(x)=\sum h_{n} x^{n}$, corresponding to the series (3), can be obtained from a simple quadratic recurrence relation:

$$
\begin{gathered}
q \cdot(n+1) \cdot(n+2) \cdot h_{n+2}=q \cdot(q-4) \cdot(3 n-1) \cdot(3 n-2) \cdot h_{n+1} \\
+2 \sum_{i=1}^{n} i \cdot(i+1) \cdot(3 n-3 i+1) \cdot h_{i+1} h_{n-i+2},
\end{gathered}
$$

Such quadratic recurrence relations are clearly a very simple and efficient way to generate power series such that the constraint that each coefficient of the series is an integer is guaranteed.

Appendix D.1. A first example corresponding to divergent series.

Consider a slight modification of the quadratic recurrence (D.1):

$$
h_{n+2}=\sum_{i=1}^{n} i \cdot(i+1) \cdot(3 n-3 i+1) \cdot h_{i+1} h_{n-i+2},
$$

Note that recurrences like (D.2) (not necessarily quadratic) obviously yield series with

integer coefficients. For instance, with the initial conditions $h_{0}=0, h_{1}=0, h_{2}=1$, the recurrence (D.2) yields the series:

$$
\begin{gathered}
x^{2}+2 x^{3}+28 x^{4}+824 x^{5}+38000 x^{6}+2424576 x^{7}+200465344 x^{8} \\
+20649137664 x^{9}+2581342891776 x^{10}+\cdots
\end{gathered}
$$

Note that this series is a divergent series. Modulo 3 this series reduces to an algebraic function:

$$
2 x^{7}+x^{5} \cdot F(x)+F(x)^{4}=0 .
$$

Modulo 5 this series reduces to an algebraic function:

$$
x^{10}+4 x^{8} \cdot F(x)+2 x^{5} \cdot F(x)^{3}+F(x)^{6}=0 .
$$

Modulo 7 this series reduces to an algebraic function of degree 48 in $x$ and degree 31 in $F(x)$.

Appendix D.2. A second example corresponding to a divergent series.

Now consider another modification of the quadratic recurrence (D.1):

$$
h_{n+2}=\sum_{i=1}^{n} i \cdot h_{i+1} h_{n-i+2},
$$

yielding the divergent series

$$
\begin{aligned}
x^{2}+x^{3} & +3 x^{4}+14 x^{5}+85 x^{6}+621 x^{7}+5236 x^{8}+49680 x^{9}+521721 x^{10} \\
& +5994155 x^{11}+74701055 x^{12}+\cdots
\end{aligned}
$$

which is a solution of the differentially algebraic equation:

$$
-x^{3}+x \cdot F(x)+F(x)^{2}-x \cdot F(x) \cdot \frac{d F(x)}{d x}=0 .
$$


If one normalizes the divergent series (D.7) by dividing through by $x^{2}$, it becomes

$$
\begin{aligned}
1+x & +3 x^{2}+14 x^{3}+85 x^{4}+621 x^{5}+5236 x^{6}+49680 x^{7}+521721 x^{8} \\
& +5994155 x^{9}+74701055 x^{10}+\cdots
\end{aligned}
$$

This normalized series is a solution of:

$$
x^{2} \cdot y(x) \cdot \frac{d y(x)}{d x}+x \cdot y(x)^{2}-y(x)+1=0 .
$$

The coefficients of (D.7) (not the ones of (D.9)) are remarkably well approximated by $c \cdot n$ ! where $c \simeq 0.21795078$. This value is deduced from 9000 coefficients of the divergent series. The Borel transform of the divergent series (D.7) is very close to

$$
\simeq \frac{0.21795078}{1-x}+0.65385 \cdot \ln (1-x) .
$$

In general the Borel transform (or the inverse Borel transform) of a differentially algebraic function is not a differentially algebraic function. However, here, the Borel transform of the divergent series (D.7) is so simple, we can imagine that it could be differentially algebraic. We have used our program on 2000 and 3000 coefficients of the Borel transform and inverse Borel transform of (D.7) and they do not seem to be differentially algebrai $\llbracket$.

Now consider perhaps the best-known example of a divergent series namely $\sum n ! \cdot x^{n}$, which is a solution of the linear ODE

$$
x^{2} \cdot \frac{d y(x)}{d x}+(x-1) \cdot y(x)+1=0 .
$$

In order to be closer to (D.9), we rewrite this equation in a quadratic way:

$$
x^{2} \cdot y(x) \cdot \frac{d y(x)}{d x}+(x-1) \cdot y(x)^{2}+y(x)=0 .
$$

One remarks that this quadratic equation (D.12) is extremely similar to the differentially algebraic equation (D.9).

Note that, again, the divergent series (D.7) reduces to algebraic functions modulo primes. Modulo 3 it reduces to the algebraic function defined by:

$$
x^{6}+2 x^{4} \cdot F(x)+x^{3} \cdot F(x)^{2}+F(x)^{4}=0 .
$$

Modulo 5 it reduces to the algebraic function defined by:

$$
\begin{gathered}
F(x)^{6}+x^{3} \cdot F(x)^{4}+2 x^{4} \cdot F(x)^{3}+x^{5} \cdot(2 x+1) \cdot F(x)^{2} \\
+2 x^{6} \cdot(x+2) \cdot F(x)+x^{8} \cdot(3 x+1)=0 .
\end{gathered}
$$

Modulo 7 it reduces to the algebraic function defined by:

$$
\begin{aligned}
F(x)^{8} & +x^{3} \cdot F(x)^{6}+2 x^{4} \cdot F(x)^{5}+3 x^{5} \cdot(x+2) \cdot F(x)^{4} \\
& +x^{6} \cdot(4 x+3) \cdot F(x)^{3}+x^{7} \cdot\left(4 x^{2}+x+1\right) \cdot F(x)^{2} \\
& +x^{8} \cdot\left(5 x^{2}+4 x+6\right) \cdot F(x)+x^{10} \cdot(1+x) \cdot(1+2 x)=0 .
\end{aligned}
$$

Note that the reduction modulo a prime of the divergent series $\sum n ! \cdot x^{n}$, in contrast, always gives polynomials. For instance modulo 7 it yields:

$$
1+x+2 x^{2}+6 x^{3}+3 x^{4}+x^{5}+6 x^{6} .
$$

|| Note that, similarly, using our program, the Borel and inverse Borel transform of Tutte's series (7) which has a non-zero radius of convergence, do not seem to be differentially algebraic.

T Which has a solution like $\exp (-1 / x) / x$. The linear ODE (D.11) can be obtained from the inverse Borel transform of $y(x)=1 /(1-x)$ using the invborel $\left((1-x) * y(x)-1, y(x),{ }^{\prime}\right.$ diffeq $\left.^{\prime}\right)$ command in Maple. 


\section{Appendix E. Algebraic functions of diagonals of rational functions}

It was noted in a previous paper (see equations (47) and (48), in Section 5.2 of 24]), that the two diagonals of rational functions $H_{1}(x)$ and $H_{2}(x)$ (12) reduce, modulo a prime, to simple algebraic functions. For instance, modulo 7, the two diagonals of rational functions (12) identify with the series expansion of the following algebraic functions:

$$
\begin{array}{r}
{ }_{2} F_{1}\left(\left[\frac{1}{2}, \frac{1}{2}\right],[1], 16 \cdot 20 \cdot x^{2}\right)=\left(1+3 x^{2}+x^{4}+6 x^{6}\right)^{-1 / 6} \\
=1+3 x^{2}+x^{4}+6 x^{6}+3 x^{14}+2 x^{16}+\cdots
\end{array}
$$

and

$$
\begin{array}{r}
{ }_{2} F_{1}\left(\left[\frac{1}{3}, \frac{1}{3}\right],[1], 27 \cdot 20 \cdot x^{2}\right)=\left(1+4 x^{2}+x^{4}\right)^{-1 / 6} \\
=1+4 x^{2}+x^{4}+4 x^{14}+2 x^{16}+\cdots
\end{array}
$$

Denote by $A_{1}$ and $A_{2}$ the two algebraic functions $\left(1+3 x^{2}+x^{4}+6 x^{6}\right)^{-1 / 6}$ and $\left(1+4 x^{2}+x^{4}\right)^{-1 / 6}$ respectively. It is natural to expect that the reduction modulo 7 of the non-holonomic series (14), namely

$$
1+4 x+3 x^{2}+3 x^{3}+x^{4}+2 x^{5}+6 x^{6}+4 x^{7}+5 x^{9}+4 x^{11}+5 x^{13}+\cdots
$$

is a solution of (13) where $H_{1}$ and $H_{2}$ are replaced by the two algebraic functions $A_{1}$ and $A_{2}$, namely solution of

$$
z^{2}-2 A_{1} \cdot z+A_{2}=0,
$$

where:

$$
\left(1+3 x^{2}+x^{4}+6 x^{6}\right) \cdot A_{1}^{6}-1=0, \quad\left(1+4 x^{2}+x^{4}\right) \cdot A_{2}^{6}-1=0 .
$$

Calculating resultants between (E.4) and the two polynomials (E.5), in order to eliminate $A_{1}$ and $A_{2}$, one gets, modulo 7 , a polynomial relation $P_{60,72}(x, z)=0$ of degree 60 in $x$ and 72 in $z$. As must be the case, this polynomial is, in fact, a function of $x^{2}$. One can check that, modulo 7, the non-holonomic series (E.3) is actually a solution of $P_{60,72}(x, z)=0$ modulo 7 .

\section{Appendix F. A simple example of the composition of two holonomic functions modulo various primes.}

Let us recall (18) the composition of the two holonomic functions (that are diagonals of rational functions) (17). The corresponding series has been shown to reduce to algebraic functions modulo 5 . Of course there is nothing special about the prime 5 . For instance, modulo 3 the series (19) for function (18) becomes

$$
\begin{aligned}
1+x+x^{2}+x^{3}+2 x^{4}+2 x^{5}+x^{6}+2 x^{7}+2 x^{8}+x^{9}+2 x^{12}+2 x^{13}+2 x^{14} \\
+2 x^{15}+x^{18}+2 x^{19}+2 x^{20}+2 x^{21}+2 x^{24}+2 x^{25}+2 x^{26}+x^{27}+\cdots
\end{aligned}
$$

which is an algebraic function given by the solution of the polynomial equation:

$$
\left(x^{2}+2 x+2\right) \cdot F(x)^{4}+2 \cdot(x+1) \cdot F(x)^{2}+2 \cdot(x+1)=0 .
$$

Modulo 7 the series (19) is, again, an algebraic function, given by the solution of the polynomial equation:

$$
\begin{gathered}
p_{36} \cdot F(x)^{36}+p_{30} \cdot F(x)^{30}+p_{24} \cdot F(x)^{24}+p_{12} \cdot\left(F(x)^{18}+F(x)^{12}\right) \\
+p_{6} \cdot\left(F(x)^{6}+1\right)=0
\end{gathered}
$$


where:

$$
\begin{aligned}
p_{36}= & x^{18}+4 x^{15}+4 x^{14}+2 x^{13}+6 x^{11}+6 x^{10}+x^{9}+2 x^{8}+2 x^{7}+3 x^{6} \\
& +4 x^{5}+2 x^{4}+5 x^{2}+2 x+6, \\
p_{30}= & 6 x^{15}+6 x^{14}+3 x^{13}+x^{12}+4 x^{11}+4 x^{10}+5 x^{9}+5 x^{8}+x^{7}+2 x^{6} \\
& +4 x^{5}+2 x^{4}+5 x^{2}+2 x+6, \\
p_{24}= & 4 x^{15}+4 x^{14}+2 x^{13}+x^{12}+x^{11}+x^{10}+4 x^{9}+6 x^{8}+3 x^{7}+4 x^{6} \\
& +4 x^{5}+2 x^{4}+5 x^{2}+2 x+6, \\
p_{12}= & 3 x^{12}+6 x^{11}+6 x^{10}+x^{9}+2 x^{8}+2 x^{7}+3 x^{6} \\
& +4 x^{5}+2 x^{4}+5 x^{2}+2 x+6, \\
p_{6}= & 6 x^{9}+4 x^{8}+6 x^{7}+4 x^{5}+2 x^{4}+5 x^{2}+2 x+6 .
\end{aligned}
$$

Modulo 11 the series (19) is yet again, an algebraic function, given by the solution of the polynomial equation:

$$
\sum_{n=0}^{n=10} q_{n} \cdot\left(F(x)^{10}\right)^{n}=0,
$$

where $q_{2}=q_{3}, q_{0}=q_{1}$ and :

$$
\begin{aligned}
q_{10} & =x^{50}+9 x^{45}+x^{44}+3 x^{43}+5 x^{42}+3 x^{41}+10 x^{40}+10 x^{39}+2 x^{37}+10 x^{36} \\
& +10 x^{35}+3 x^{34}+10 x^{32}+9 x^{31}+3 x^{30}+10 x^{29}+7 x^{28}+9 x^{27}+8 x^{26} \\
& +3 x^{25}+4 x^{24}+4 x^{23}+5 x^{21}+6 x^{20}+4 x^{19}+8 x^{18}+9 x^{17}+7 x^{16}+10 x^{15} \\
& +7 x^{13}+7 x^{12}+8 x^{10}+3 x^{9}+8 x^{8}+x^{6}+5 x^{5}+2 x^{4}+2 x^{3}+x^{2}+2 x+10, \\
q_{9} & =2 x^{45}+10 x^{44}+8 x^{43}+6 x^{42}+8 x^{41}+8 x^{40}+5 x^{39}+x^{37}+5 x^{36}+3 x^{35} \\
& +10 x^{34}+2 x^{33}+3 x^{32}+x^{31}+7 x^{30}+8 x^{28}+x^{27}+x^{25}+3 x^{24}+5 x^{22} \\
& +9 x^{21}+9 x^{20}+4 x^{19}+4 x^{18}+2 x^{17}+8 x^{16}+8 x^{15}+2 x^{14}+9 x^{13}+9 x^{12} \\
& +6 x^{11}+7 x^{10}+3 x^{9}+8 x^{8}+x^{6}+5 x^{5}+2 x^{4}+2 x^{3}+x^{2}+2 x+10, \\
q_{8} & =6 x^{45}+8 x^{44}+2 x^{43}+7 x^{42}+2 x^{41}+4 x^{40}+2 x^{39}+7 x^{37}+2 x^{36} \\
& +4 x^{35}+2 x^{34}+9 x^{33}+4 x^{32}+2 x^{31}+2 x^{30}+2 x^{29}+6 x^{28}+x^{27}+6 x^{26} \\
& +7 x^{25}+9 x^{24}+5 x^{22}+10 x^{21}+2 x^{20}+4 x^{19}+5 x^{18}+x^{17}+5 x^{16}+3 x^{15} \\
& +7 x^{14}+3 x^{13}+3 x^{12}+10 x^{11}+10 x^{10}+3 x^{9}+8 x^{8}+x^{6}+5 x^{5} \\
& +2 x^{4}+2 x^{3}+x^{2}+2 x+10 \\
q_{7} & =2 x^{40}+9 x^{39}+4 x^{37}+9 x^{36}+6 x^{35}+5 x^{34}+3 x^{33}+6 x^{32}+10 x^{31} \\
& +10 x^{30}+10 x^{29}+4 x^{27}+8 x^{26}+4 x^{25}+7 x^{24}+7 x^{23}+10 x^{22}+x^{21} \\
& +8 x^{20}+4 x^{19}+10 x^{18}+7 x^{17}+x^{16}+10 x^{14}+6 x^{13}+6 x^{12}+8 x^{11} \\
& +3 x^{10}+3 x^{9}+8 x^{8}+x^{6}+5 x^{5}+2 x^{4}+2 x^{3}+x^{2}+2 x+10
\end{aligned}
$$




$$
\begin{aligned}
q_{6} & =(x+1)^{2} \cdot\left(10 x^{38}+3 x^{37}+6 x^{36}+5 x^{35}+7 x^{34}+3 x^{33}+2 x^{32}+5 x^{31}\right. \\
& +10 x^{30}+6 x^{28}+9 x^{27}+4 x^{26}+7 x^{25}+x^{24}+2 x^{23}+x^{22}+2 x^{21}+9 x^{20} \\
& +8 x^{19}+8 x^{18}+2 x^{17}+x^{16}+2 x^{14}+3 x^{13}+6 x^{12}+6 x^{11}+3 x^{10}+8 x^{9} \\
& \left.+4 x^{8}+9 x^{7}+8 x^{6}+8 x^{5}+10 x^{4}+10 x^{3}+5 x^{2}+4 x+10\right), \\
q_{5} & =2 x^{35}+8 x^{34}+9 x^{33}+2 x^{32}+9 x^{31}+x^{30}+3 x^{29}+2 x^{28}+2 x^{27} \\
& +9 x^{26}+x^{25}+7 x^{24}+6 x^{23}+3 x^{22}+8 x^{21}+8 x^{20}+4 x^{19}+4 x^{18}+2 x^{17} \\
& +8 x^{16}+8 x^{15}+2 x^{14}+9 x^{13}+9 x^{12}+6 x^{11}+7 x^{10}+3 x^{9}+8 x^{8}+x^{6} \\
& +5 x^{5}+2 x^{4}+2 x^{3}+x^{2}+2 x+10 \\
q_{4} & =(x+1) \cdot\left(x^{34}+3 x^{33}+7 x^{32}+5 x^{31}+5 x^{30}+8 x^{29}+7 x^{28}+x^{27}\right. \\
& +6 x^{26}+6 x^{25}+8 x^{24}+10 x^{23}+4 x^{22}+7 x^{20}+x^{19}+3 x^{18}+5 x^{17} \\
& +4 x^{16}+3 x^{15}+7 x^{14}+4 x^{13}+3 x^{12}+4 x^{11}+7 x^{10}+x^{9}+2 x^{8}+6 x^{7} \\
& \left.+5 x^{6}+7 x^{5}+9 x^{4}+4 x^{3}+9 x^{2}+3 x+10\right), \\
q_{3} & =5 x^{30}+x^{29}+5 x^{28}+9 x^{27}+3 x^{26}+9 x^{25}+10 x^{24}+4 x^{23}+6 x^{21} \\
& +10 x^{20}+4 x^{19}+9 x^{18}+8 x^{17}+4 x^{16}+5 x^{15}+5 x^{14}+x^{13}+x^{12} \\
& +4 x^{11}+3 x^{9}+8 x^{8}+x^{6}+5 x^{5}+2 x^{4}+2 x^{3}+x^{2}+2 x+10, \\
q_{1} & =10 x^{25}+8 x^{24}+5 x^{23}+7 x^{22}+6 x^{21}+5 x^{20}+4 x^{19}+6 x^{17} \\
& +9 x^{16}+6 x^{15}+4 x^{14}+x^{11}+6 x^{10}+3 x^{9}+8 x^{8}+x^{6}+5 x^{5} \\
& +2 x^{4}+2 x^{3}+x^{2}+2 x+10 .
\end{aligned}
$$

More generally, one can conjecture that, modulo a prime $p$, the corresponding polynomial equation will be of the form:

$$
\sum_{n=0}^{p-1} q_{n} \cdot\left(F(x)^{p-1}\right)^{n}=0 .
$$

\section{Appendix G. Symmetries of the differentially algebraic equation (26).}

Recall $P\left(x, F(x), F^{\prime}(x), F^{\prime \prime}(x), F^{\prime \prime \prime}(x), F^{(4)}(x)\right)=0$, i.e. the polynomial corresponding to equation (26) for the differentially algebraic function (18) given by the composition of two holonomic functions. It has been seen that if $F(x)$ is a solution of (26), $A \cdot F(x)$ is also a solution of (26). In fact this scaling symmetry is a consequence of a more general symmetry of the polynomial equation (26). If one considers the two solutions of the order-two linear differential operator $(16 x-1) \cdot x \cdot D_{x}^{2}+(32 x-1) \cdot D_{x}+4$, namely $S_{1}(x)=H_{1}(x)$ (see (17)) and $S_{2}(x)=\ln (x) \cdot H_{1}(x)+\mathcal{H}_{1}(x)$ where the power series $\mathcal{H}_{1}(x)$ reads:

$$
\begin{array}{r}
8 x+84 x^{2}+\frac{2960}{3} x^{3}+\frac{37310}{3} x^{4}+\frac{820008}{5} x^{5}+\frac{11153912}{5} x^{6}+\frac{1086209696}{35} x^{7} \\
+\frac{3074289075}{7} x^{8}+\frac{396822097100}{63} x^{9}+\frac{28763739153292}{315} x^{10}+\frac{73453759289456}{55} x^{11} \\
+\frac{9740489140942196}{495} x^{12}+\frac{375490923772997200}{1287} x^{13}+\cdots
\end{array}
$$

One finds that not only is $S_{1}\left(H_{2}(x)\right)$ a solution of the non-linear ODE (27), but $S_{2}\left(H_{2}(x)\right)$ is also a solution of the non-linear ODE (27), and more generally, any 
linear combination $A \cdot S_{1}\left(H_{2}(x)\right)+B \cdot S_{2}\left(H_{2}(x)\right)$ is a solution of (27). This is a general result: namely that for compositions of holonomic functions, the differentially algebraic equation inherits the (differential Galois) symmetries of the linear differential operator corresponding to the first holonomic function.

\section{Appendix H. Other solutions of the differentially algebraic equation (26).}

Appendix H.1. Movable singularities for the differentially algebraic equation (26).

Among the miscellaneous solutions of (27) one has a one-parameter family such as:

$$
\begin{aligned}
1+ & 4 v \cdot x+4 v \cdot(9 v+4) \cdot x^{2}+16 v \cdot\left(25 v^{2}+18 v+9\right) \cdot x^{3} \\
& +4 v \cdot\left(1225 v^{3}+1200 v^{2}+792 v+400\right) \cdot x^{4} \\
& +16 v \cdot\left(3969 v^{4}+4900 v^{3}+3900 v^{2}+2448 v+1225\right) \cdot x^{5} \\
& +16 v \cdot\left(53361 v^{5}+79380 v^{4}+73500 v^{3}+53200 v^{2}+32166 v+15876\right) \cdot x^{6} \\
& \quad+\cdots
\end{aligned}
$$

which reduce to the series (19) for $v=1$. The radius of convergence, $R$, of that series is clearly a function of the parameter $v(R \rightarrow 0$ when $v \rightarrow \infty)$. One clearly has movable singularities.

For $v=2$ the series (H.1) reads:

$$
\begin{aligned}
1 & +8 x+176 x^{2}+4640 x^{3}+132672 x^{4}+3981600 x^{5}+123476480 x^{6} \\
& +3921615488 x^{7}+126825870848 x^{8}+4160174803232 x^{9}+138026770667008 x^{10} \\
& +4622425971834496 x^{11}+156011453811509760 x^{12}+5300311971681413248 x^{13} \\
& +181089920155530497536 x^{14}+6217365460907222370816 x^{15}+\cdots \quad \text { (H.2) }
\end{aligned}
$$

This series has a radius of convergence $R \simeq 0.02722469$ corresponding to a singularity at $x_{c} \simeq 0.02722469$.

Appendix H.2. Reduction to algebraic functions modulo primes for the other solutions of two differentially algebraic equation (26).

The $v=2$ series (H.2) is a solution of the non-linear differential equation (27), but we have no idea if it can be seen as a composition of holonomic functions, and in particular a composition of diagonals of rational functions. Modulo 3 this series (H.2) becomes

$$
\begin{aligned}
1+ & 2 x+2 x^{2}+2 x^{3}+2 x^{6}+2 x^{7}+2 x^{8}+2 x^{9}+x^{10}+x^{11}+x^{13}+x^{14} \\
& +2 x^{16}+2 x^{17}+2 x^{18}+2 x^{21}+2 x^{24}+2 x^{25}+2 x^{26}+2 x^{27}+\cdots
\end{aligned}
$$

which can be seen to be an algebraic solution, modulo 3 , of

$$
\left(x^{2}+2 x+2\right) \cdot F(x)^{4}+2 \cdot(x+1) \cdot F(x)^{2}+2 \cdot(x+1)=0,
$$

which is the same polynomial equation as (F.2) (even if the series (F.1) and (H.3) are different). Modulo 5 the series (‥2) becomes

$$
\begin{gathered}
1+3 x+x^{2}+2 x^{4}+3 x^{7}+3 x^{8}+2 x^{9}+3 x^{10}+x^{11}+3 x^{13}+x^{14}+x^{15}+3 x^{16} \\
+4 x^{17}+x^{18}+2 x^{20}+4 x^{21}+x^{22}+4 x^{23}+x^{26}+4 x^{27}+\cdots
\end{gathered}
$$


which similarly satisfies, modulo 5 the algebraic equation

$$
\begin{aligned}
\left(x^{8}+\right. & \left.x^{6}+4 x^{5}+2 x^{4}+3 x^{3}+3 x^{2}+3 x+1\right) \cdot F(x)^{16} \\
& +\left(x^{4}+3 x^{3}+3 x^{2}+3 x+1\right) \cdot\left(F(x)^{12}+F(x)^{4}+1\right) \\
& +\left(x^{2}-x+1\right)\left(3 x^{4}+5 x^{3}+6 x^{2}+4 x+1\right) \cdot F(x)^{8}=0,
\end{aligned}
$$

which is actually the same polynomial equation as (25) (even if the series (21) and (H.5) are different). Modulo 7 the series (ㅍ.2) becomes

$$
\begin{aligned}
1+ & x+x^{2}+6 x^{3}+x^{4}+x^{6}+5 x^{8}+6 x^{10}+2 x^{12}+2 x^{13}+5 x^{14}+4 x^{15} \\
& +6 x^{16}+2 x^{17}+2 x^{17}+6 x^{18}+3 x^{19}+6 x^{20}+3 x^{21}+4 x^{22} \\
& +6 x^{23}+5 x^{24}+3 x^{25}+\cdots
\end{aligned}
$$

which similarly satisfies, modulo 7 the same algebraic equation as (F.3). Modulo 11 the series (H.2) can actually be seen to be an algebraic function, modulo 11, of the same polynomial equation as (F.5).

One can expect the series (- $(\mathrm{H} .2)$ to reduce to algebraic functions modulo every prime or power of a prime, but not necessarily with the same polynomial equations when the primes become large enough.

Remark: Actually the series (H.1) seems to reduce to algebraic functions modulo every prime or power of a prime for many prime-integer values of $v$, even many rational values of $v$, with the polynomial equations also reducing to the previous polynomials (25), (F.2), (F.3), (F.5).

Such simple results provide a strong incentive to systematically study the reduction, modulo primes or powers of a prime, of all solution-series with integer coefficients of differentially algebraic equations.

\section{Appendix I. Linear diff-Padé approach of a differentially algebraic function: fixed singularities versus movable singularities.}

The traditional diff-Padé approach amounts to seeking linear differential equations with polynomial coefficients. However, from a more formal (and less well-defined) viewpoint, if one seeks linear differential equations with no longer polynomial functions but holonomic function coefficients, it is easy to see that the (differentially algebraic) function (18) can also be seen as a solution of the following linear second order differential operator but with transcendental coefficients

$$
\begin{aligned}
\frac{d p(x)}{d x} \cdot p(x) \cdot(1-16 p(x)) \cdot D_{x}^{2} & \\
- & \left(p(x) \cdot(1-16 p(x)) \cdot \frac{d^{2} p(x)}{d x^{2}}-(1-32 p(x)) \cdot\left(\frac{d p(x)}{d x}\right)^{2}\right) \cdot D_{x} \\
& \quad-4 \cdot\left(\frac{d p(x)}{d x}\right)^{3},
\end{aligned}
$$

where the pullback $p(x)$ is the transcendental function:

$$
p(x)=x \cdot{ }_{2} F_{1}\left(\left[\frac{1}{2}, \frac{1}{2}\right],[1], 16 x\right) .
$$

It is quite easy to verify that (19), the series expansion of the differentially algebraic function (18), is actually a solution of the formal transcendental linear differential 
equation (I.1). The singularities of (I.1) are fixed, and correspond to the head coefficient, namely:

$$
\frac{d p(x)}{d x} \cdot p(x) \cdot(1-16 p(x))=0 .
$$

At first sight one expects an infinite number of zeros from the vanishing condition (1.3) of transcendental functions. Here, it seems that the only solutions of (1.3) (fixed singular points of (I.1) ) are 0 and the previously given value $x \simeq 0.04602833718455$.

Keeping this very formal result in mind, let us display the kind of result we obtained for a "traditional" linear diff-Padé approximant analysis (the coefficients are now polynomials). Recall that near $x \simeq 1 / 16,16 x \cdot{ }_{2} F_{1}([1 / 2,1 / 2],[1], 16 x)$ behaves like $p(x) \simeq-16 x \cdot \ln (1-16 x) / 2$, and the (differentially algebraic) function (18) behaves like ${ }_{2} F_{1}([1 / 2,1 / 2],[1], p(x))$ with $p(x) \rightarrow \infty$, namely like $\ln (-p(x)) / 2 /(-p(x))^{1 / 2}$, which is an irregular singularity.

The order four and eight differential approximants give a singularity extremely close to the previous exact value with an exponent very close to zero (.005353572).

One also finds many singularities very close to $1 / 16$, namely .062500000019 $\pm 1.32710^{-11} .06249973, .0624999976, .062499999808, \ldots$ the exponents being all over the place and changing from approximant to approximant. This type of behaviour of differential approximants is characteristic of an irregular singularity.

\section{References}

[1] L. Onsager, Crystal Statistics. I. A Two-Dimensional Model with an Order-Disorder Transition, Phys. Rev. 65, (1944) 117-149.

[2] G. M. Viswanathan The hypergeometric series for the partition function of the 2-D Ising model, JSTAT 2015, P07004 arXiv:1411.2495v2 [cond-math;stat-mech].

[3] C. N. Yang The Spontaneous Magnetization of a Two-Dimensional Ising Model, Phys. Rev. 85, (1952) 808-816.

[4] T. T. Wu, B. M. McCoy, C. A. Tracy and E. Barouch, Spin-spin correlation functions for the two-dimensional Ising model: Exact theory in the scaling region, Phys. Rev. B 13 (1976) 316-374.

[5] W. P. Orrick, B. G. Nickel, A. J. Guttmann and J. H. H. Perk, The Susceptibility of the Square Lattice Ising Model: New Developments, J. Stat. Phys. 103, (2001) 795-841.

[6] B. Nickel, On the singularity structure of the 2D Ising Susceptibility, J. Phys. A 32: Math. Gen. (1999) 3889-3906

[7] N. Zenine, S. Boukraa, S. Hassani and J-M. Maillard, The Fuchsian differential equation of the square-lattice Ising model $\chi^{(3)}$ susceptibility, J. Phys. A: Math. Gen. 37 (2004) 9651-9668, arXiv:math-ph/0407060

[8] N. Zenine, S. Boukraa, S. Hassani and J-M. Maillard, Square lattice Ising model susceptibility: connection matrices and singular behavior of $\chi^{(3)}$ and $\chi^{(4)}$, J. Phys. A: Math. Gen. 38 (2005) 9439-9474, arXiv:math-ph/0506065

[9] S. Boukraa, A. J. Guttmann, S. Hassani, I. Jensen, J-M. Maillard, B. Nickel and N. Zenine, Experimental mathematics on the magnetic susceptibility of the square lattice Ising model, J. Phys. A: Math. Theor. 41 (2008) 45520 (51 pp), arXiv:0808.0763

[10] B. Nickel, I. Jensen, S. Boukraa, A. J. Guttmann, S. Hassani, J-M. Maillard and N. Zenine, Square lattice Ising model $\tilde{\chi}^{(5)}$ ODE in exact arithmetics, J. Phys. A: Math. Theor. 42 (2010) 195205 (24 pp), arXiv:1002.0161 v2 [math-ph].

[11] A. Bostan, S. Boukraa, A. J. Guttmann, S. Hassani, I. Jensen, J-M. Maillard and N. Zenine, High order Fuchsian equations for the square-lattice Ising model: $\tilde{\chi}^{(5)}$, J. Phys. A: Math. Theor. 42 (2009) 275209 (32 pp), arXiv:0904.1601 v1 [math-ph].

[12] S. Boukraa, S. Hassani, I. Jensen, J-M. Maillard and N. Zenine, High order Fuchsian equations for the square lattice Ising model: $\chi^{(6)}$, J. Phys. A: Math. Theor. 43 (2010) 115201 (22 pp), arXiv:0912.4968v1 [math-ph]. 
[13] Y-B Chan, A. J. Guttmann, B. G. Nickel and J. H. H. Perk, The Ising Susceptibility Scaling Function, J. Stat. Phys. 145, 549-590, (2011) arXiv:1012.5272 3

[14] S. Boukraa, S. Hassani, J-M. Maillard, B. M. McCoy, J-A. Weil, N. Zenine, Painlevé versus Fuchs, J. Phys. A 39 (2006) 12245-12263 Special issue for the centenary of the publication of the Painlevé VI equation, http://arxiv.org/pdf/math-ph/0602010 v3

[15] A. J. Guttmann and J-M. Maillard, Automata and the susceptibility of the square lattice Ising model modulo powers of primes, J. Phys. A: Math. Theor. 42 (2015) 474001 Special issue for R.J. Baxter, arXiv:1507.02872 v2 [math-ph].

[16] J. Pantone, GuessFunc: Automatically forming conjectures about differentially algebraic power series. (Forthcoming.)

[17] K. G. Binmore, Analytic functions with Hadamard gaps, Bull. London Math. Soc, 1 (1969), 211-217

[18] M. Weiss and G. Weiss, On the Picard property of lacunary power series, Studia Math. 22 $(1962 / 63), 221-245$

[19] C. Pommerenke, Some examples of power series with non-Hadamard gaps, Math. Zeit. 187 (2):165-170 May (1984).

[20] A. Bostan, S. Boukraa, G. Christol, S. Hassani and J-M. Maillard, Ising n-fold integrals as diagonals of rational functions and integrality of series expansions, J. Phys. A: Math. Theor. 46 (2013) 185202 (44 pp), arXiv:1211.6645v2 [math-ph].

[21] L. Lipshitz, The diagonal of a D-finite power series is D-finite, J. Algebra, 113 (1988), 373-378.

[22] H. Furstenberg, Algebraic functions over finite fields, J. Algebra 7, (1967) 271-277.

[23] G. Christol, Fonctions algébriques modulo n, submitted to Contemporary Mathematics (2016)

[24] S. Boukraa and J-M. Maillard, Selected non-holonomic functions in lattice statistical mechanics and enumerative combinatorics, J. Phys. A: Math. Theor. 49 (2016) Special issue for A. J. Guttmann arXiv:1510.04651 2 [math-ph].

[25] W. T. Tutte, Chromatic solutions. II , Canad. J. Math. 34, (1982) 952-960.

[26] W. T. Tutte, Map-colourings and differential equations, In Progress in graph theory, Waterloo, Ont. 1982, Academic Press, Toronto, (1984) 477-485.

[27] C. G. J. Jacobi, Über die Differentialgleichung, welcher die Reihen $1 \pm 2 q \pm 2 q^{4} \pm 2 q^{9}+\ldots$. Genüge Leisten, Journal für die reine und angewandte Mathematik, 36 97-112 (1848) Crelle's Journal

[28] S. Chakravarty and M. Ablowitz, Parameterizations of the Chazy equation, Stud. Appl. Math. 124, Issue 2, 105-135, (2010), arXiv:0902.3468 1[nlin.SI]"

[29] A. Cobham, On the base-dependence of sets of numbers recognizable by finite automata, Math. Systems Theory, (1969) 3, 186-192.

[30] G. Christol, Diagonals of Rational Fractions, Newsletter of the Europ. Math. Soc. (2015), Issue 97, 37-43.

[31] Z. Mebkhout, Structure de Frobenius et Exposants de la Monodromie p-adique des Equations différentielles, Contemp. Math. 474 (2008) 175-245.

[32] L. Lipshitz and L. A. Rubel, A gap theorem for power series solutions of differentially algebraic equations, Amer. J. Math. 108, (1986) 1193-1214.

[33] F. Bergeron and C. Reutenauer, Combinatorial Resolution of Systems of Differential equations III: Special Class of Differentially Algebraic Series, Europ. J. Comb. 11 (1990) 501-512.

[34] A. M. Odlyzko and L. B. Richmond, A differential equation arising in chromatic sum theory, In Proceedings of the fourteenth Southeastern conference on combinatorics, graph theory and computing (Boca Raton, Fla. 1983) 40, 263-275, 1983.

[35] O. Bernardi and M. Bousquet-Mélou, Counting colored planar maps: algebraicity results, J. Combin. Theory, B 101 (5), (2011) 315-377.

[36] S. Boukraa, S. Hassani, J-M. Maillard, B. M. McCoy, J-A. Weil, N. Zenine, Fuchs versus Painlevé, J. Phys. A 40 (2007), 2583-2614 http://arxiv.org/pdf/math-ph/0701014 v2

[37] J. Chazy, Sur les équations différentielles dont l'intégrale générale est uniforme et admet des singularités essentielles mobiles, C.R. Acad. Sc. Paris 149, (1909) 563-565.

[38] J. Chazy, Sur les équations différentielles du troisième ordre et d'ordre supérieur dont l'intégrale a ses points critiques fixes, Acta Math. 34 (1911), no. 1, 317-385.

[39] V. Ovsienko and S. Tabachnikov, What is the Schwarzian derivative?, Notices of the AMS, 56, Number 1, 34-36

[40] B. Osgood, Old and New on the Schwarzian derivative, 275-308, Springer Verlag (1998) in Quasiconformal Mappings and Analysis, A collection of Papers honoring F.W. Gehring.

[41] A. Bostan, S. Boukraa, S. Hassani, M. van Hoeij, J-M. Maillard, J-A. Weil and N. Zenine, The Ising model: from elliptic curves to modular forms and Calabi-Yau equations, J. Phys. A: Math. Theor. 44 (2011) 045204 (44 pp), arXiv:1007.0535 v1. 
[42] L. Lipshitz and A. J. van der Poorten, 1988, Rational Functions, Diagonals, Automata and Arithmetic, (Dedicated to the memory of Kurt Mahler), in Number Theory: Proceedings of the First Conference of the Canadian Number Theory Association, 339-358, Edited by R.A Mollin, de Gruyter, 1990.

[43] J. Denef and L. Lipshitz, Algebraic Power Series and Diagonals, J. Numb. Theor. 26, (1985) 46-67.

[44] B. Adamczewski and J. P. Bell, Diagonalization and Rationalization of Algebraic Laurent Series, Annales Scientifiques de l'ENS 46, fascicule 6 (2013), 963-1004.

[45] I. Jensen, http://www.ms.unimelb.edu.au/ iwan/ising/Ising_ser.html

[46] D. A. Cox, J. Little and D. O'Shea, Ideals, varieties, and algorithms. An introduction to computational algebraic geometry and commutative algebra, Fourth edition. Undergraduate Texts in Mathematics. Springer, Cham, 2015. xvi+646 ISBN: 978-3-319-16720-6; 978-3-31916721-3

[47] J. H. H. Perk, Quadratic identities for Ising model correlations, Phys. Lett. 79 A, (1980) 3-5.

[48] J. H. H. Perk, Nonlinear partial difference equations for Ising model n-point Green's functions, Proc. II International Symposium on Selected Topics in Statistical Mechanics, Dubna, August 25-29, 1981, JINR, Dubna, USSR, (1981) 138-151.

[49] B. M. McCoy, J. H. H. Perk and T. T. Wu, Ising field theory: quadratic difference equations for the n-point Green's functions on the square lattice, Phys. Rev. Lett. 46, (1981) 757-760.

[50] O. Bernardi and M. Bousquet-Mélou, Counting colored planar maps: differential equations, (2015), arXiv: 1507.02391v2 [math.CO].

[51] M. Bousquet-Mélou and A. Jehanne, Polynomial equations with one catalytic variable, algebraic series and map enumeration, J. Combin. Theory, Ser. B 96 (2006) 623-672.

[52] M. Bousquet-Mélou and J. Courtiel, Spanning forests in regular planar maps, J. Comb. Theor. A 135, (2015) 1-59, arXiv:1306.4536 1 [math.CO].

[53] W. T. Tutte, Chromatic sums for rooted planar triangulations. III. The case $\lambda=3$, Canad. J. Math. 25, (1973) 780-790.

[54] W. T. Tutte, Chromatic sums for rooted planar triangulations. The cases $\lambda=1$ and $\lambda=2$, Canad. J. Math. 25, (1973) 426-447.

[55] W. T. Tutte, Chromatic sums for rooted planar triangulations. V. Special equations, Canad. J. Math. 26, (1974) 893-907.

[56] E. Maillet, Sur les séries divergentes et les équations différentielles, Ann. Scient. de l'ENS, 3ème série, tome 20, (1920) 487-518.

[57] Y. André, Arithmetic Gevrey series and transcendence. A survey. Proc. J. Arith. de Lille, Journal de Théorie des Nombres de Bordeaux, 15, (2003) 1-10.

[58] G. Pólya, Sur les séries entières dont la somme est une fonction algébrique, Enseignement Math. 22, (1921-22) 38-47.

[59] G. Pólya and G Szegö, Problems and Theorems in Analysis II, Springer-Verlag, (1976) 